\title{
Uncovering the local inelastic interactions during manufacture of ductile cast iron: How the substructure of the graphite particles can induce residual stress concentrations in the matrix
}

Andriollo, Tito; Hellström, Kristina; Sonne, Mads Rostgaard; Thorborg, Jesper; Tiedje, Niels Skat; Hattel, Jesper Henri

Published in:

Journal of the Mechanics and Physics of Solids

Link to article, DOI:

10.1016/j.jmps.2017.11.005

Publication date:

2018

Document Version

Peer reviewed version

Link back to DTU Orbit

Citation (APA):

Andriollo, T., Hellström, K., Sonne, M. R., Thorborg, J., Tiedje, N. S., \& Hattel, J. H. (2018). Uncovering the local inelastic interactions during manufacture of ductile cast iron: How the substructure of the graphite particles can induce residual stress concentrations in the matrix. Journal of the Mechanics and Physics of Solids, 111, 333-357. https://doi.org/10.1016/j.jmps.2017.11.005

\section{General rights}

Copyright and moral rights for the publications made accessible in the public portal are retained by the authors and/or other copyright owners and it is a condition of accessing publications that users recognise and abide by the legal requirements associated with these rights.

- Users may download and print one copy of any publication from the public portal for the purpose of private study or research.

- You may not further distribute the material or use it for any profit-making activity or commercial gain

- You may freely distribute the URL identifying the publication in the public portal 


\section{Uncovering the local inelastic interactions during manufacture of ductile cast iron: How the substructure of the graphite particles can induce residual stress concentrations in the matrix}

Tito Andriollo ${ }^{\mathrm{a}, \mathrm{d}}$, Kristina Hellström ${ }^{\mathrm{b}}$, Mads Rostgaard Sonne ${ }^{\mathrm{a}}$, Jesper Thorborg ${ }^{\mathrm{a}, \mathrm{c}}$, Niels Tiedje $^{\mathrm{a}}$ and Jesper Hattel ${ }^{\mathrm{a}}$

a Department of Mechanical Engineering, Technical University of Denmark, DK-2800 Kgs. Lyngby, Denmark

${ }^{\mathrm{b}}$ Department of Materials \& Manufacturing, School of Engineering, Jönköping University, 55111 Jönköping, Sweden

c MAGMA GmbH, D-52072 Aachen, Germany

${ }^{\mathrm{d}}$ Corresponding author. Address: Technical University of Denmark, Produktionstorvet, Building 425, room 225, 2800 Kgs. Lyngby, Denmark. Phone: +45 452547 22. E-mail: titoan@mek.dtu.dk

\section{Abstract}

Recent X-ray diffraction (XRD) measurements have revealed that plastic deformation and a residual elastic strain field can be present around the graphite particles in ductile cast iron after manufacturing, probably due to some local mismatch in thermal contraction. However, as only one component of the elastic strain tensor could be obtained from the XRD data, the shape and magnitude of the associated residual stress field have remained unknown. To compensate for this and to provide theoretical insight into this unexplored topic, a combined experimental-numerical approach is presented in this paper. First, a material equivalent to the ductile cast iron matrix is manufactured and subjected to dilatometric and high-temperature tensile tests. Subsequently, a two-scale hierarchical topdown model is devised, calibrated on the basis of the collected data and used to simulate the interaction between the graphite particles and the matrix during manufacturing of the industrial part considered in the XRD study. The model indicates that, besides the viscoplastic deformation of the matrix, the effect of the inelastic deformation of the graphite has to be considered to explain the magnitude of the XRD strain. Moreover, the model shows that the large elastic strain perturbations recorded with XRD close to the 
graphite-matrix interface are not artifacts due to e.g. sharp gradients in chemical composition, but correspond to residual stress concentrations induced by the conical sectors forming the internal structure of the graphite particles. In contrast to common belief, these results thus suggest that ductile cast iron parts cannot be considered, in general, as stressfree at the microstructural scale.

\section{Keywords}

thermomechanical processes $\mathrm{A}$; residual stress $\mathrm{B}$; inhomogeneous material $\mathrm{B}$; elasticviscoplastic material B; Cast iron; Graphite;

\section{Contents}

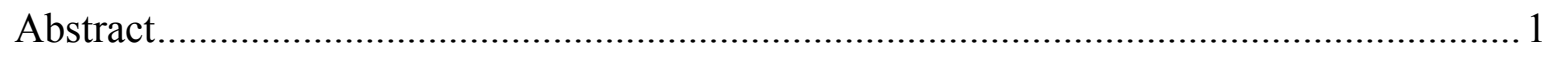

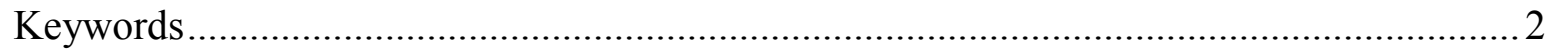

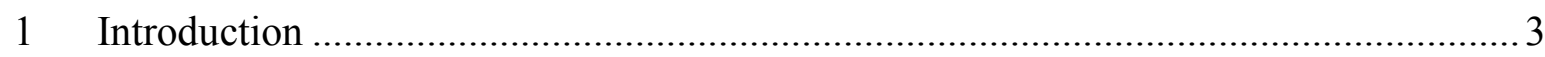

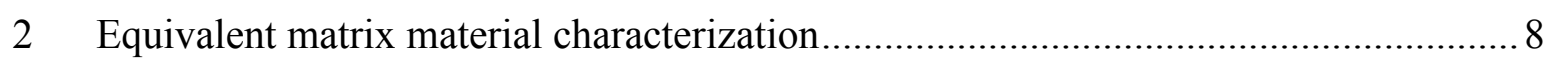

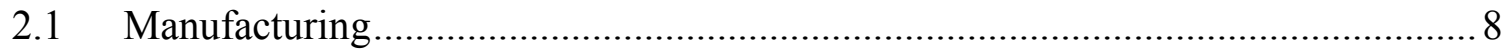

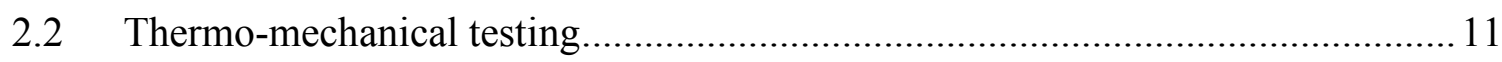

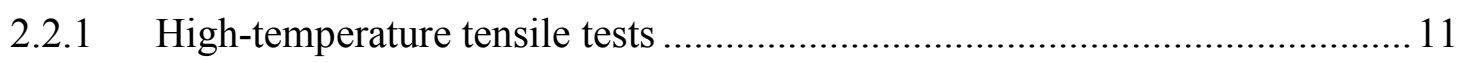

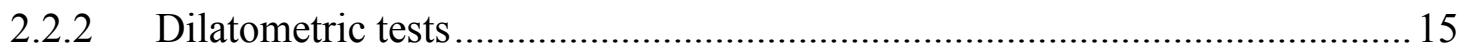

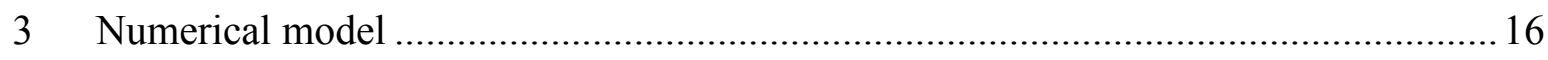

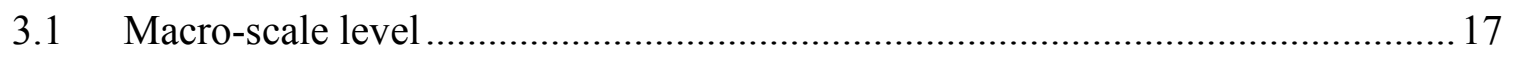

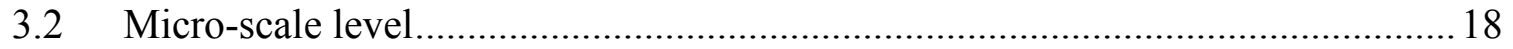

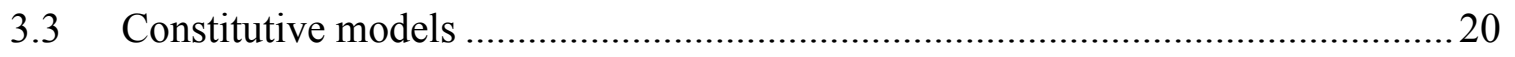

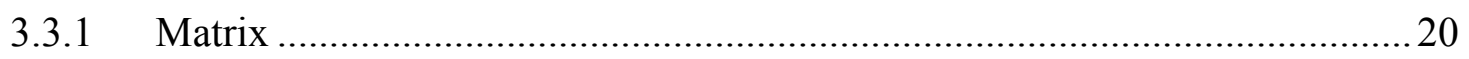

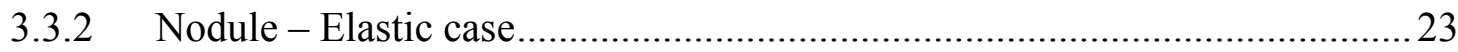

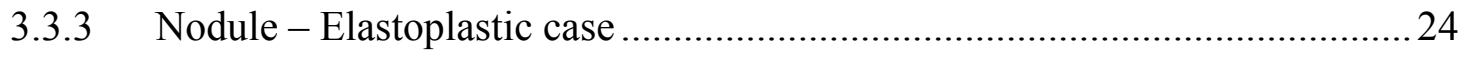

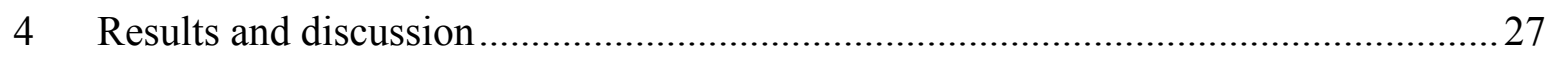

4.1 Predicted VS measured residual elastic strain in the matrix .................................2 27

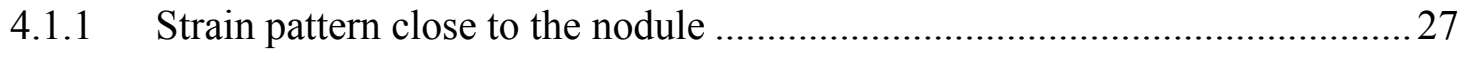

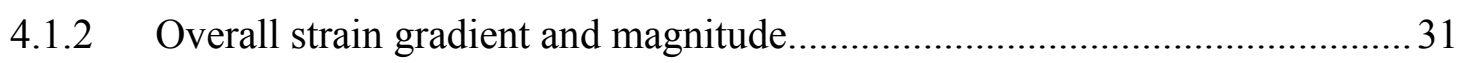


4.2 Predicted residual stress in the matrix 38

5 Conclusions 41

Appendix A: Materials parameters identification for Peric's model 43

Appendix B: Implicit integration scheme for Merkle's model....................................... 45

Appendix C: Materials parameters identification for Hoffman's model........................... 47

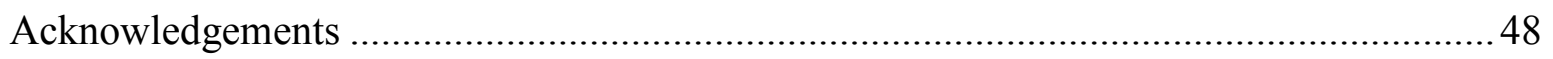

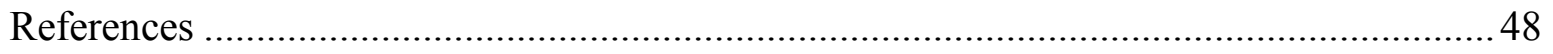

\section{Introduction}

The discovery of ductile cast iron, also referred to as nodular cast iron or spheroidal graphite iron (SGI), dates back to 1943, when Keith D. Millis of the International Nickel Company Research Laboratory found out that a small addition of magnesium to gray cast iron resulted in the formation of spherical graphite particles (Loper Jr., 1994). In the subsequent decades, the material has experienced an enormous and constantly growing development (Labrecque and Gagne, 1998), certified by the fact that today, more than 70 years after its introduction, it represents as much as $25 \%$ of the total castings produced worldwide (47th Census of World Casting Production, 2013). The main reason behind this phenomenal success is the unique combination of castability, high ductility and strength this material can offer, along with lower prices compared to traditional low carbon steels (Ductile Iron Society, 2013). This makes SGI the best choice for manufacturing small and medium sized heavily loaded parts with high demands for consistent quality for the automotive industry and very large industrial components with extreme demands for mechanical properties, particularly fatigue strength and fracture toughness (Tiedje, 2010). Due to the primary technological importance that SGI has in our society, and the huge monetary losses associated with premature failure of critical SGI components in the automotive and energy sectors, major efforts have been devoted in the past years to understand the fracture mechanisms under both monotonic and cyclic loading, as reported in a recent review article (Hütter et al., 2015a). In this respect, a major challenge is represented by the material microstructure, which, in contrast to many other metallic alloys, is not homogeneous but consists of spherical graphite particles, called spheroids or nodules, 
embedded in a metallic matrix which can be either ferritic, pearlitic or a mixture of the two (Grimvall, 1997)(Sjögren and Svensson, 2004). The impact of this composite nature at the micro-scale on the material fracture behavior has been documented by fracture tests conducted on ferritic and ferritic-pearlitic SGI, which have clearly shown that the crack propagation path is strongly affected by the presence of the graphite nodules (Baer, 2014)(Di Cocco et al., 2014)(Fernandino and Boeri, 2015) (Iacoviello and Di Cocco, 2016). The consequence is that the fracture process of SGI can hardly be explained on the basis of standard theoretical models which consider the material as homogeneous (Dong et al., 1997)(Berdin et al., 2001). Prompted by the recent progress in advanced characterization techniques such as micro X-ray tomography, which allows unprecedented insight into the morphology and distribution of the microstructural phases, more advanced models which take into account the local interactions arising between the different microstructural elements are now emerging, whose development is a current research area (Costa et al., 2010)(Collini and Pirondi, 2014)(Hütter et al., 2015b)(Salomonsson and Olofsson, 2017)(Fernandino et al., 2017).

Concerning this last point, recent research by the present authors has shown that, despite most of the models proposed so far consider SGI as stress-free at the microstructural scale, significant interactions between the graphite nodules and the matrix occur during the manufacturing process, leading to a localized residual stress state in the material. The reason is the larger thermal contraction experienced by the matrix during solid-state cooling compared to the nodules, with the former that tends to compress the latter in a hydrostatic fashion. This aspect was often neglected in the past, probably due to the scarce knowledge of the mechanical properties of the nodules, pointed out in (Andriollo and Hattel, 2016), and the technical difficulties of performing direct residual stress measurement around embedded particles whose diameter does not normally exceed $150 \mu \mathrm{m}$. In the last 10-15 years however, such experimental limitations have been progressively disappearing due to the advent of new, powerful X-ray diffraction techniques based on synchrotron radiation, which have dramatically increased the possibilities of characterizing the material stress state at the micro-scale (Yang et al., 2002)(Yang et al., 2004). Indeed, by exploiting one of these new techniques, known as differential-aperture X-ray microscopy (DAXM), the 
existence of a non-negligible residual elastic strain field in the ferrite matrix region surrounding the nodules has recently been shown for the first time in a thin sample extracted from an industrial SGI component (Zhang et al., 2016). In this respect, it is worth remarking that the reduced size of the sample and the sub-surface location of the nodules selected for the measurements have allowed ruling out the hypothesis that the recorded elastic strain field could be associated to either a macroscopic residual stress field or to surface-related effects. Moreover, the gradient of the recorded elastic strain has been found globally consistent with that expected on the basis of the type of thermo-mechanical interaction between the nodules and the matrix described above. Altogether, these results are an indicator that the stress-free assumption may be inadequate to describe the roomtemperature microstructure of SGI.

Unfortunately, despite showing unambiguously that the nodules and the matrix interact mechanically during manufacturing, the findings of (Zhang et al., 2016) do not provide a complete picture of the residual elastic strain field existing around the nodules, nor of the associated residual stress field, which would be essential for developing any advanced SGI fracture theory. The reason is twofold and can be better appreciated with the help of figure 1. First of all, only one component of the elastic strain tensor, corresponding to the normal strain $\varepsilon_{F F}^{e l}$ along the F-axis of the F-H coordinate system of figure 1 (a), was measured,

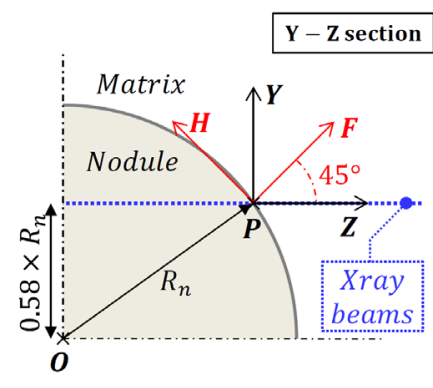

(a)

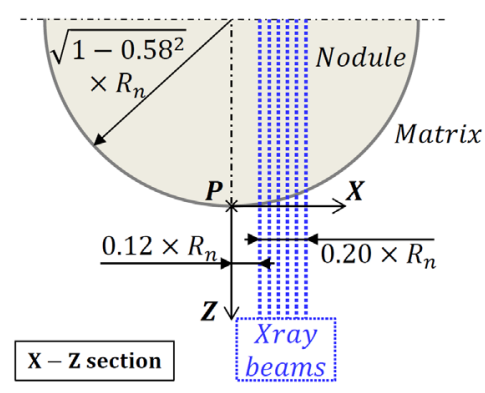

(b)

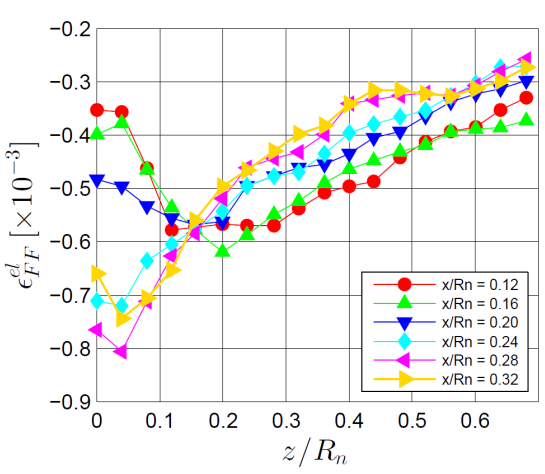

(c)

Figure 1 - DAXM measurements of residual elastic strain in the ferrite matrix close to nodule " $A$ " in (Zhang et al., 2016). (a) - (b) Schematic of the X-ray beam direction, indicated with blue dots, with respect to the nodule. The XHF coordinate system is $45^{\circ}$ rotated about the $\mathrm{X}$-axis compared to the XYZ system. Note that the $\mathrm{Y}-\mathrm{Z}$ section crosses the nodule center, denoted with the letter "O". $\mathrm{R}_{\mathrm{n}}=25 \mu \mathrm{m}$ is the nodule radius. (c) Normal residual elastic strain in the F-direction, measured along the 6 blue dotted lines parallel to the Z-axis depicted in (b). 
which is not sufficient to derive the full stress tensor via the generalized Hooke's law. Secondly, the variation of this single strain component was recorded along straight parallel paths, depicted with blue dots in figure 1 (b), whose non-radial orientation complicates significantly the interpretation of the results. To compensate for these issues, one possibility is to construct a theoretical model which simulates the relevant physics and allows predicting both the full residual elastic strain and stress fields around the nodules. If the model is capable of reproducing the experimentally recorded elastic strain within engineering accuracy, the linearity of Hooke's law should guarantee that the model can be used to estimate the stress with a similar level of accuracy.

A first, partial attempt to apply the strategy just described to estimate the residual stress field around the graphite particles was made in (Zhang et al., 2016), on the basis of the linear elastic micro-mechanical unit cell model proposed in (Andriollo et al., 2016b) to simulate the thermo-elastic properties of ferritic SGI at room temperature. Despite being able to explain the global tendency of $\varepsilon_{F F}^{e l}$ to become less compressive with increasing values of the $\mathrm{z}$-coordinate (see figure 1), the analysis based on this simplified model was affected by deficiencies so severe that it could not be used to make any sound conclusions regarding the residual stress field. The first reason was that the overall gradient of $\varepsilon_{F F}^{e l}$ and its maximum compressive values were overestimated by approximately a factor of 2 , probably due to the model incapability of accounting for inelastic deformation of the SGI matrix during solid-state cooling, which was clearly observable in the ferrite grains neighboring the nodules (Zhang et al., 2016). Regarding this, it is worth mentioning that some simulations were performed allowing time-independent plastic deformation in the matrix via the standard J2-flow theory, using yield stress data proper to pure ferrite. Nevertheless, the model predictions did not change considerably, suggesting that, not surprisingly, the deformation of the matrix during cooling is probably controlled by timedependent relaxation mechanisms. The second reason that made this early analysis inadequate was the incapability of reproducing, and thereby providing a valid explanation for, the non-monotonic profiles of $\varepsilon_{F F}^{e l}$ visible in figure 1 (c) when the distance from the nodule is below $\approx 20 \%$ of the nodule's radius. More generally, it was not possible to clarify whether such strain perturbations were due to some mechanical effect or were 
simply a reflection of sharp gradients in chemical composition, which are known to affect the value of the unstrained lattice spacing used as reference to calculate the experimental strain (Levine et al., 2015). It has to be emphasized that this last issue is particularly critical when considered in the overall context of the present discussion, as the large amplitude of the $\varepsilon_{F F}^{e l}$ perturbations (up to $\approx 5 \times 10^{-4}$ ) might be associated with large variations of the residual stress field around the nodules $\left(5 \times 10^{-4 *} \mathrm{E} \approx 103 \mathrm{MPa}\right.$, with $\mathrm{E}=205 \mathrm{GPa}$ being the isotropic ferrite Young's modulus), which might possibly play a decisive role in the fracturing process of SGI.

Given the serious deficiencies of the early analysis outlined above, a new attempt to quantify the residual stress field existing around the graphite particles is described in this paper. In detail, a new model which simulates both the elastic and inelastic interactions occurring between the nodules and the matrix during manufacturing of the ferritic SGI component considered in the DAXM study is introduced and used to 1) justify the overall magnitude and gradient of the residual elastic strain measured in the matrix, 2) explain the nature of the residual elastic strain perturbations recorded in proximity to the nodule-matrix interface, 3) provide a reasonable estimate of the associated residual stress field, to be used as future basis for improving the current knowledge of the mechanical and fracture behavior of SGI.

It should be clear, from what stated previously, that a prerequisite for the success of such model is the availability of data regarding the time-dependent deformation of the ferrite matrix over a large range of temperatures. Due to its peculiar chemical composition, rich in silicon and low in carbon, which somewhat resembles that of the so-called electrical steels (Malagari, 1973)(Moses, 1990), comprehensive information is lacking in the literature. To the authors' best knowledge, steady-state creep data can be found, but not full uniaxial stress-strain curves or measurements of the thermal expansion coefficient in conditions other than room temperature. Therefore, Section 2 of this article will be dedicated to report the results of an experimental campaign aimed at collecting these data, whereas the presentation of the model and of the related numerical findings will be given in Section 3 and Section 4 respectively. 


\section{Equivalent matrix material characterization}

The problem of obtaining proper thermo-mechanical data for the SGI matrix can be split into two separate tasks. The first one involves re-creating an alloy with the same characteristics of the material surrounding the nodules, henceforth called equivalent matrix material. The second one concerns testing such alloy to obtain values for those properties which are not available in the literature, but are necessary to calibrate a thermo-mechanical rate-dependent constitutive model.

\subsection{Manufacturing}

In order to manufacture the equivalent matrix material, it is necessary to define its meaning in more precise, quantitative terms. To this end, it is convenient to review briefly the solidification process experienced by a SGI with composition equivalent to that considered in the DAXM experiments performed by (Zhang et al., 2016). This will also help in understanding the numerical model that will be presented later in Section 3.

Under the assumption of thermodynamic equilibrium, which should suffice for the present purposes, it may be assumed that solidification of SGI proceeds as dictated by the red dashed arrow reported on the phase diagram of figure 2. The first step is eutectic solidification in the strictest sense, which involves in sequence 1) independent nucleation of the nodules and of the austenite in the melt, 2) envelopment of the nodules by the austenite dendrites, with their further growth which becomes controlled by carbon diffusion from the liquid through the austenite, and 3) eventual solidification of the liquid remaining between the different dendrites or in between the secondary arms of the same dendrite (Rivera et al., 2002). The second step is solid-state-cooling in the austenitic field, which is characterized by carbon diffusion from the austenite to the graphite, associated with the progressive reduction in solubility of this element in the metallic phase. According to (Di Cocco et al., 2013), this causes the radius of the nodules to increase from $90 \%$ of the value at room temperature, which represents the size of such particles by the end of the eutectic solidification, up to approximately $95 \%$. The third step starts when the upper inter-critical temperature $\mathrm{T}_{\text {ui }}$ of $811^{\circ} \mathrm{C}$ is reached and the eutectoid reaction begins: the austenite loses carbon and transforms into ferrite, causing the nodules to expand to almost their final size 


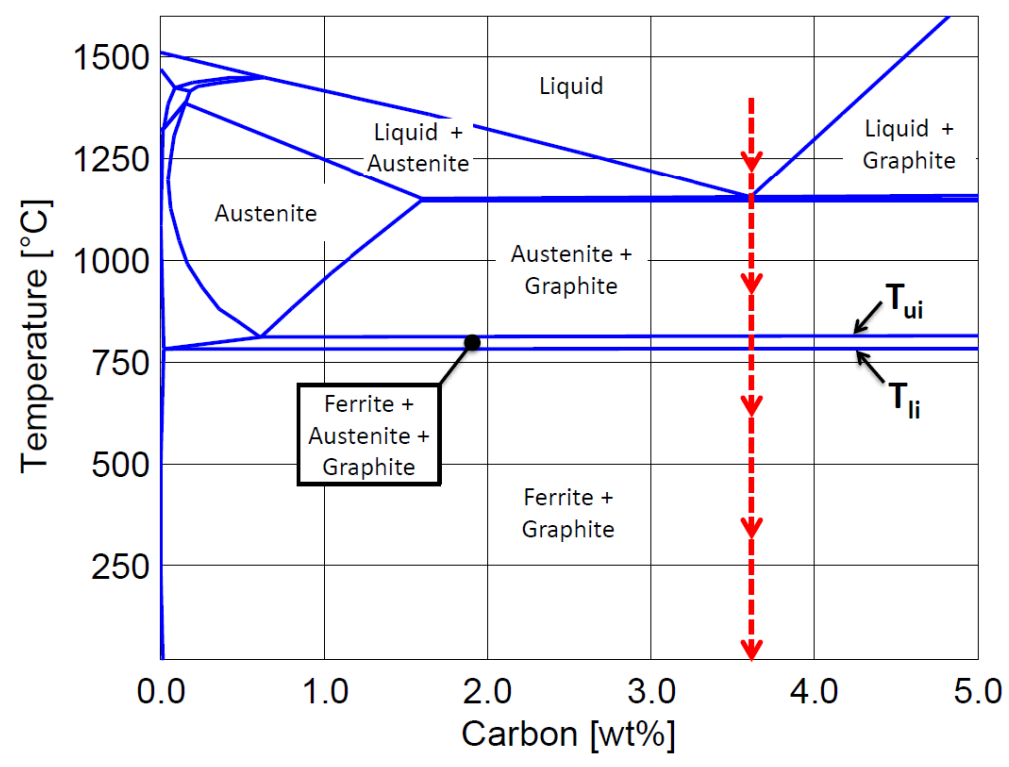

Figure 2 - Slice of the multidimensional phase diagram corresponding to the chemical composition of the SGI used in the analysis of (Zhang et al., 2016). Data generated using Thermo-Calc (Andersson et al., 2002).

(Di Cocco et al., 2013). Finally, the fourth step involves solid-state cooling in the ferritic field from the lower inter-critical temperature $\mathrm{T}_{\text {li }}$ of $792{ }^{\circ} \mathrm{C}$ down to room temperature, and entails only minor changes in chemistry.

What should be gleaned from the previous description is that the matrix composition changes continuously during cooling. Both the silicon and another important alloying element like manganese are rejected by the nodules, so that their average concentration in the matrix can be assumed almost constant. But the carbon concentration varies over a few orders of magnitude according to the solubility limit in austenite first, and then in ferrite. This means that one should, in principle, manufacture a number of equivalent matrix materials equal to the number of temperatures at which mechanical testing is needed, each with a different chemical composition. As this would require a huge investment of time and resources, the choice in this work was to investigate only the properties over the temperature range spanned by the ferritic field. On the one hand, this allows working with only one alloy, as the matrix composition does not vary significantly in this range. On the other hand, as will be explained in Section 3, the ferritic field corresponds to the part of the cooling process where the magnitude of the stresses forming around the nodules becomes 
significant, making the accurate knowledge of the material's behavior more important in this stage compared to e.g. solid state cooling in the austenitic field. Consequently, the equivalent matrix material was defined, from a chemical perspective, as the alloy having the same average composition of the SGI used in (Zhang et al., 2016) except for a carbon content in the range of the solubility limit in the ferritic field.

Manufacturing of the equivalent matrix material was carried out by melting iron blocks with purity level of $99.9 \%$ in an induction furnace and adjusting the silicon and carbon content by addition of ferrosilicon $75 \mathrm{wt} \%$ and graphite powder. The melt was poured at a temperature of $\approx 1600{ }^{\circ} \mathrm{C}$ into sand molds to produce castings of the geometry shown in figure 3 (a). Optical microscope examinations revealed that the final microstructure was, as expected, entirely ferritic. The chemical composition was checked further via optical emission spectrometry and it is reported in table 1. It can be observed that the concentration of $\mathrm{Si}$ and $\mathrm{Mn}$ was $1.99 \mathrm{wt} \%$ and $0.10 \mathrm{wt} \%$, respectively. These values are very common for the SGI grades used in the wind energy sector, due to the requirements of ferritic structure and low-temperature ductility (Stefanescu, 2017). However, with respect to the particular SGI considered in (Zhang et al., 2016), whose composition is listed for comparison in the same table 1 , the concentration of $\mathrm{Si}$ and $\mathrm{Mn}$ is underestimated by $13 \%$ and $55 \%$, respectively. In relation to this, it is worth remarking that both elements give rise to micro-segregation during solidification of SGI. Particularly, their final concentration tends to be higher - for $\mathrm{Si}$ - and lower - for $\mathrm{Mn}$ - in the region around the graphite nodules (Selig and Lacaze, 2000)(Vazehrad et al., 2015), which is clearly the most important when it comes to analyzing the nodule-matrix interaction. As a consequence, a Mn level in the equivalent matrix material lower than the average value in SGI should not be seen as detrimental in the context of the present work. Concerning $\mathrm{Si}$, its concentration in the $\mathrm{Si}$ rich regions of SGI is normally only $\approx 10 \div 15 \%$ larger than the average value (Selig and Lacaze, 2000). This means that the Si content close to the graphite nodules considered in (Zhang et al., 2016) might reasonably be $\approx 0.5 \mathrm{wt} \%$ larger than the Si content in the equivalent matrix material manufactured in the present work. This difference is not expected to have a large impact on the mechanical properties, especially at temperatures where creep becomes important (Davies, 1963). Nevertheless, its potential influence on the 
model predictions in terms of the residual stress and strain field around the nodules will be assessed via the uncertainty analysis carried out in section 4.1.2.
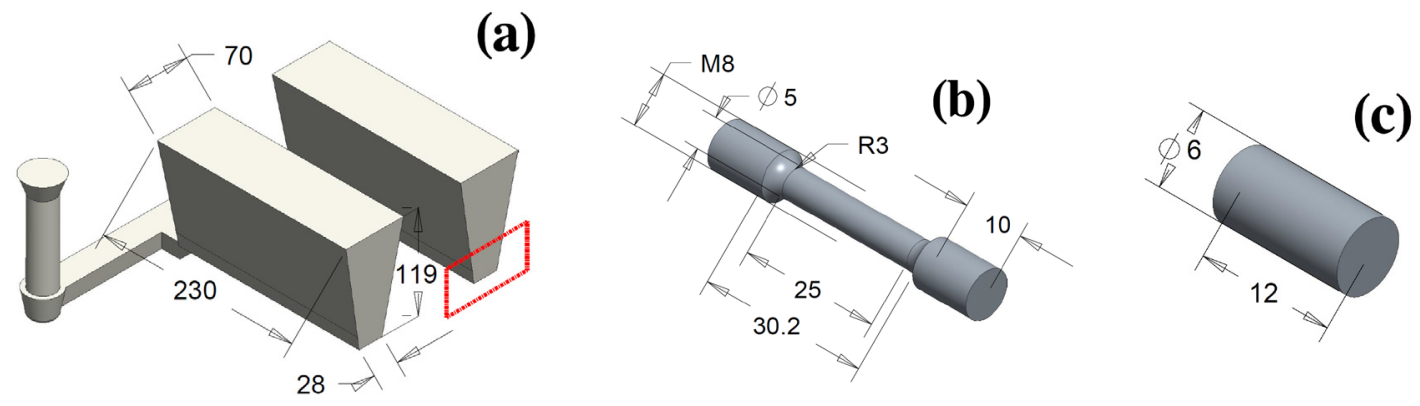

Figure 3 - Geometry of the casting (a), of the high-temperature tensile test samples (b) and of dilatometric samples (c) used to characterize the equivalent matrix material. Units are millimeters.

Table 1 - Comparison between the chemical composition of the SGI used in (Zhang et al., 2016) and that of the equivalent matrix material manufactured in the present work. Values are expressed as wt $\%$.

\begin{tabular}{ccccccccccc}
\hline & $\mathrm{C}$ & $\mathrm{Si}$ & $\mathrm{Mn}$ & $\mathrm{P}$ & $\mathrm{S}$ & $\mathrm{Cr}$ & $\mathrm{Ni}$ & $\mathrm{Co}$ & $\mathrm{Cu}$ & $\mathrm{Ti}$ \\
\hline SGI & 3.68 & 2.30 & 0.22 & 0.015 & 0.011 & 0.027 & 0.048 & 0.024 & 0.016 & 0.017 \\
$\begin{array}{c}\text { Equivalent matrix } \\
\text { material }\end{array}$ & 0.036 & 1.99 & 0.10 & 0.007 & 0.005 & 0.029 & 0.021 & 0.005 & 0.018 & 0.008 \\
\hline
\end{tabular}

\subsection{Thermo-mechanical testing}

\subsubsection{High-temperature tensile tests}

As shortly mentioned in the introduction, experimental studies are available in the literature regarding steady-state creep of ferritic alloys with composition similar to that of the equivalent matrix material defined in this work. The most relevant for the present analysis are summarized in table 2, with indication of the specific silicon concentration and

Table 2 - Experimental steady-state creep studies performed on ferritic alloys with composition similar to that of the equivalent matrix material considered in the present work.

\begin{tabular}{lll}
\hline Authors and year & Silicon level (wt\%) & Temperature level $\left({ }^{\circ} \mathrm{C}\right)$ \\
\hline (Davies, 1963) & $1.04 \div 5.07$ & $390 \div 586$ \\
(Barrett and Nix, 1965) & 3.0 & 643 \\
(Barrett, 1967) & 3.0 & $820 \div 1025$ \\
(Stang et al., 1973) & 3.0 & $1100 \div 1405$ \\
\hline
\end{tabular}


temperature range investigated.

Steady-state creep data alone is, however, not sufficient to characterize completely the time-dependent behavior of the equivalent matrix material, but needs to be supported by e.g. uniaxial tensile tests conducted at different strain rates, as discussed in (Pina et al., 2014) for the case of pearlitic steels. For this reason, cylindrical dog-bone-shaped specimens with the geometry shown in figure 3 (b) were machined from the bottom region of the castings, marked with a red dashed rectangle in figure 3 (a), and tested in tension at temperatures between 20 and $700{ }^{\circ} \mathrm{C}$. The tests were carried out using the Gleeble 1500 thermo-mechanical simulator, which, as described in detail elsewhere (Brown et al., 1997)(Zhang et al., 2010), allows direct heating of the specimen via AC current flowing through the water-cooled copper clamps. Each test was performed in three steps: 1) heating of the specimen at $10{ }^{\circ} \mathrm{C} / \mathrm{s}$ up to the prescribed temperature, 2) application of $60 \mathrm{~s}$ holding time to reach steady-state thermal conditions, 3) straining of the specimen in displacement control mode. During this last stage, determination of the stress and strain was made on the basis of the applied force and of the radial contraction measured at the center of the specimen via a $\mathrm{C}$-shaped gauge, which is part of the standard Gleeble equipment.

The tensile test results for different combinations of temperature and nominal strain rate are reported in figure 4 (a) and (b). It has to be remarked that the nominal strain rate, which was kept fixed during each test, is defined on the basis of the clamps' displacement. In contrast, the plotted strain and strain rate refer to the deformation experienced by the central cross-section of the specimen, which is the location where the thermocouple controlling the temperature was located. This explains the non-flat appearance of the curves shown in figure 4 (a) and their vertical shift compared to the nominal strain value they are associated with. Focusing the attention on figure 4 (b), it can be noted that the equivalent matrix material shows some strain rate sensitivity already at $350{ }^{\circ} \mathrm{C}$, which, not surprisingly, becomes more pronounced at $700{ }^{\circ} \mathrm{C}$. This is in agreement with the common rule of thumb that creep mechanisms for metals start at approximately $35 \%$ of the melting temperature in Kelvin (Ashby et al., 2010), which corresponds to $\approx 340{ }^{\circ} \mathrm{C}$ in the present case. Moreover, this fact provides also an explanation for why the simulations mentioned in 


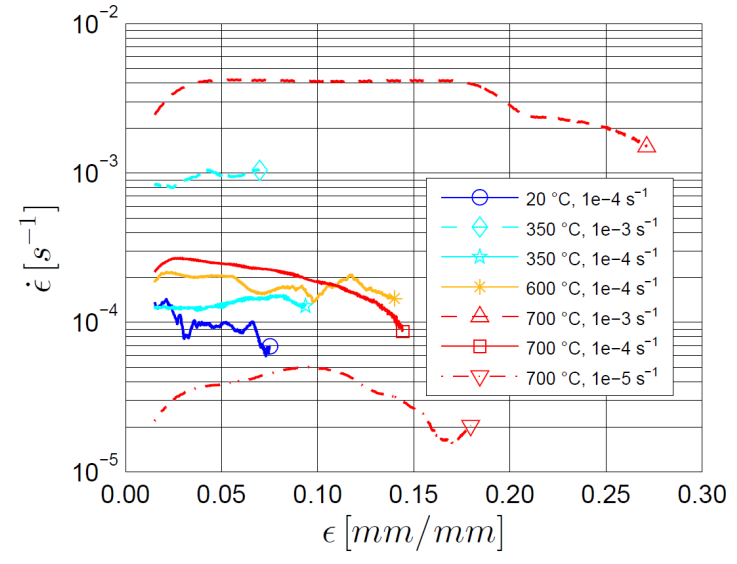

(a)

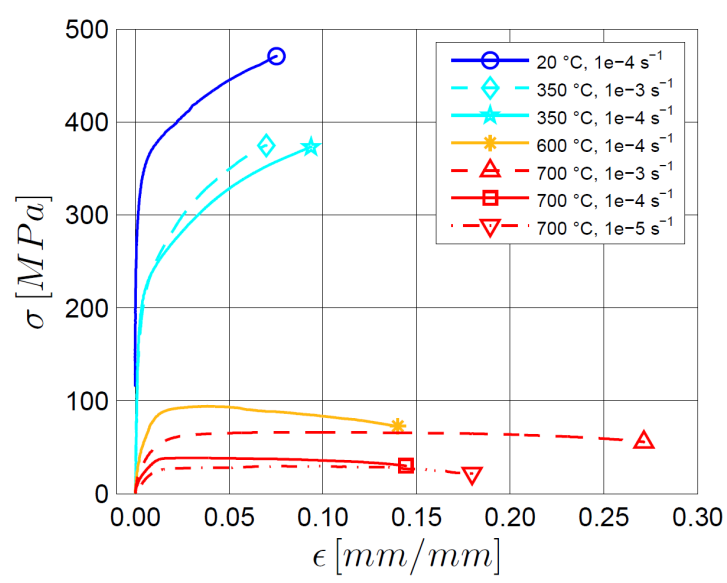

(b)

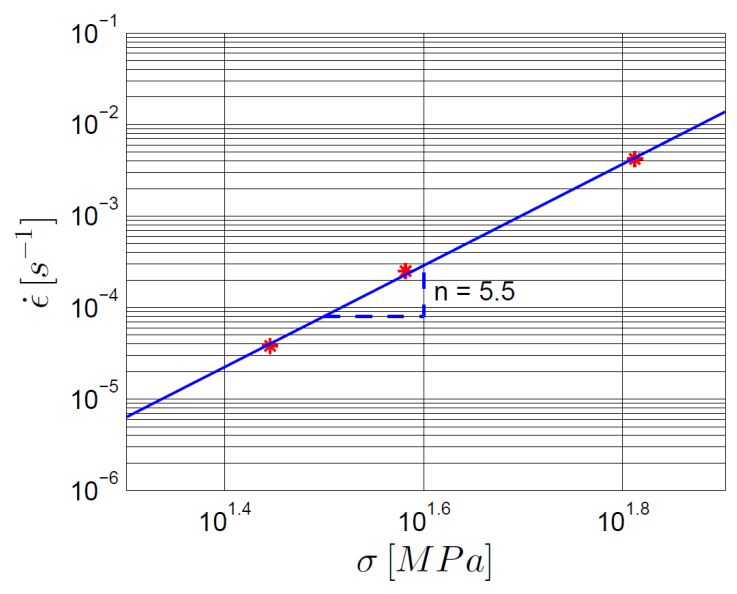

(c)

Figure 4 - Equivalent matrix material tested in uniaxial tension at different temperatures and nominal strain rates. (a) Strain rate vs strain curves. (b) Stress vs strain curves. (c) Log-log plot of the strain rate at $\varepsilon=5 \%$ for the curves at $700{ }^{\circ} \mathrm{C}$ as a function of the corresponding stress. In all graphs, the engineering definition of stress and strain applies.

the second-last paragraph of the introduction, based on rate-independent models for the matrix, were unsuccessful.

An important point to be discussed is the sensitivity of the results to the grain size. The reason is that the ferrite grains observed around the graphite nodules in the DAXM experiments of (Zhang et al., 2016) had an average size of $\approx 30$ microns, whereas those of the equivalent matrix material tested in this work are about ten times larger. In this respect, it should be noted that in the curves at $700{ }^{\circ} \mathrm{C}$ it is possible to identify plateau regions 
where the stress and the strain rate are approximately constant. These conditions correspond to those defining steady-state creep, which can be described by the generalized law (Mukherjee, 2002)

$$
\dot{\varepsilon}_{s s}=A \frac{D G b}{k T}\left(\frac{b}{d}\right)^{p}\left(\frac{\sigma}{G}\right)^{n}
$$

where $\dot{\varepsilon}_{S S}$ is the creep rate, $D$ is the approximate diffusivity, $G$ is the shear modulus, $b$ is the Burger vector, $k$ is the Boltzmann constant, $T$ is the test temperature, $d$ is the grain size, $p$ is the grain size exponent, $\sigma$ is the flow stress and $n$ is the strain rate sensitivity. The stress and strain rates corresponding to the plateau regions can then be identified with $\sigma$ and $\dot{\varepsilon}_{S S}$ in the equation above. By plotting these quantities on a log-log plot and performing a linear fit (figure 4 (c)), a value of 5.5 for the exponent $n$ is obtained. According to the discussion reported in (Walser and Sherby, 1982) and (Mukherjee, 2002), the combination of this value of $n$, the coarse material microstructure and the strain rates measured in the tests, suggests that the material deformation is controlled by dislocation climb. This is in agreement with the findings of (Davies, 1963), who came to the same conclusion by analyzing the steady-state creep rate of Fe-Si alloys with $\mathrm{Si}$ between 1 and $5 \mathrm{wt} \%$ at temperatures between 475 and $600{ }^{\circ} \mathrm{C}$. Remarkably, for this particular type of creep the exponent $p$ in equation (1) is zero, meaning that the grain size does not affect, to a first approximation, the deformation mechanism (Walser and Sherby, 1982)(Schneibel and Heilmaier, 2014). Consequently, the curves recorded at $700{ }^{\circ} \mathrm{C}$ should look similar to those that would be obtained for a material with the same chemical composition, but smaller grains. This should hold true for the curves at 350 and 600 degrees as well, as they lie above the critical homologous temperature of 0.35 required for dislocation climb (Ashby et al., 2010). Concerning the room temperature case instead, (Suits and Chalmers, 1961) performed uniaxial tensile tests on ferrite with $3 \mathrm{wt} \%$ silicon and grain sizes between 20 and 170 microns. They observed that the stress-strain curves differed substantially in the initial yield stress and in the maximum elongation, but not in the hardening behavior up to $\approx 8 \%$ strain. As the deformation that is expected to occur during manufacturing of SGI is likely to be within this limit, it seems reasonable to assume that the curves associated with different grain sizes differ only by a shift along the stress axis, whose magnitude depends 
on the difference in yield stress. This can be quantified by means of the Hall-Petch relation as

$$
\Delta \sigma_{y}=k_{p}\left(\frac{1}{\sqrt{d_{1}}}-\frac{1}{\sqrt{d_{2}}}\right)
$$

where $d_{1}$ and $d_{2}$ denote the grain sizes and $k_{p}$ is the Petch constant. A value of 0.70 $\mathrm{MPa} \cdot \mathrm{m}^{1 / 2}$ was found for $k_{p}$ in (Suits and Chalmers, 1961), which was used in the present investigations to adjust the room temperature stress-strain curve to the desired grain size. It is worth remarking that for standard mild steel with negligible silicon content the value of $k_{p}$ is about $0.74 \mathrm{MPa} \cdot \mathrm{m}^{1 / 2}$ (Smith and Hashemi, 2006).

\subsubsection{Dilatometric tests}

Besides obtaining a more precise quantification of its time-dependent behavior at high temperature, another important reason for manufacturing the equivalent matrix material was to measure its thermal contraction during cooling, which plays a crucial role in the formation of the type of micro-scale stresses discussed in the introduction. Indeed, values of the coefficient of thermal expansion (CTE) for ferrite up to $1000{ }^{\circ} \mathrm{C}$ are available in the literature (Bonora and Ruggiero, 2005), but the effect of silicon levels comparable to those considered in the present study do not appear to have been documented yet. To investigate this point, cylindrical specimens as shown in figure 3 (c) were machined from the bottom region of the cast wedge blocks depicted in figure 3 (a), and subjected to dilatometric analysis. A push-rod dilatometer, model 402C, from Netzsch was used for the purpose. Heating and cooling rates were set to $8 \mathrm{~K} / \mathrm{min}$ and the maximum temperature to $1273 \mathrm{~K}$ $\left(1000^{\circ} \mathrm{C}\right)$. An isothermal time of 15 minutes was added at maximum temperature, before cooling. Before testing the samples the temperature program was performed on an aluminum oxide standard, with the same measures as the samples, in order to obtain a standard curve. Subsequent measurements were then corrected by the standard curve. Both the standard and the samples were subjected to the same treatment and tested in helium atmosphere. The load of the push rod on the samples (and standard) was $25 \mathrm{cN}$. The logged data on temperature and length variation were exported and used to calculate the contraction experienced by the equivalent matrix material during cooling from a generic 
temperature $\mathrm{T}$ down to $20^{\circ} \mathrm{C}$. The results are shown in figure $\mathbf{5}$, where they are compared with the measurements conducted on another alloy which was $50 \%$ higher in silicon. It can be seen that a small effect of this element on the material thermal contraction can be detected, even though it is so limited that it can be neglected for the present purposes.

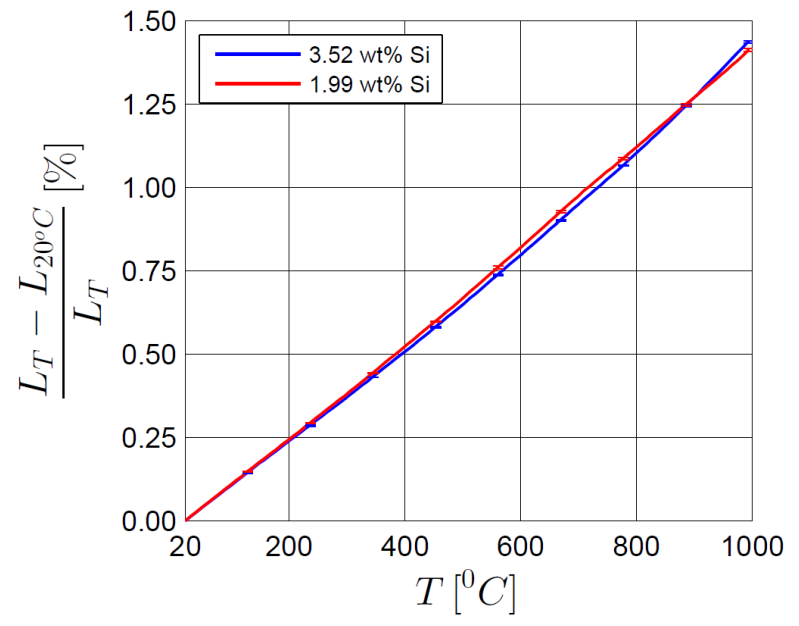

Figure 5 - Engineering thermal strain experienced by the equivalent matrix material upon cooling from a temperature $\mathrm{T}$ to $20^{\circ} \mathrm{C}$. The small error bars indicate the standard deviation recorded by repeating the measurement 3 times.

\section{$3 \quad$ Numerical model}

A two-scale hierarchical top-down model is used to study the thermo-mechanical interaction between the nodules and the matrix during manufacturing. First, solidification and solid-state cooling of the entire SGI component is simulated at the macro-scale with a continuum-type model. Subsequently, the temperature vs time curve at the location from which the DAXM sample was extracted is passed on to the micro-scale level, where the single microstructural constituents of SGI are modelled as independent entities. The following three subsections provide separate descriptions of the models adopted at both scales and of the constitutive relations used for the nodules and for the matrix. 


\subsection{Macro-scale level}

The sample used in the DAXM study of (Zhang et al., 2016) was extracted from a large wind turbine main shaft cast in a metal mold. The geometry of the casting and the location from which the sample was taken are sketched in figure 6 (a).

To simulate the manufacturing process of the main shaft at the macroscopic level the software MAGMA5 (MAGMA GmbH, 2017) is used. This numerical code, widely adopted in the foundry industry, solves the energy and linear momentum equations in a coupled way to determine the temperature and displacement fields during solidification and solid-state cooling. Effects like visco-elasto-plastic material behavior, varying heat transfer coefficient at the casting-mold interface and latent heat release associated with phase transformations can all be accounted for. A thorough discussion of MAGMA5, its features and the specific setup used to simulate the casting of the main shaft of figure 6 (a) is outside the scope of the present paper, but can be found in (Sonne et al., 2017). Here, only the results strictly relevant to the current analysis are reported. These consist in the numerically-predicted cooling curve relative to the position from which the DAXM sample was extracted, which is shown in figure $6(b)$.

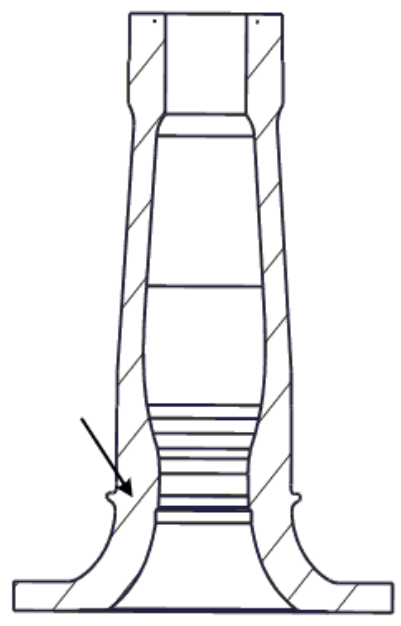

(a)

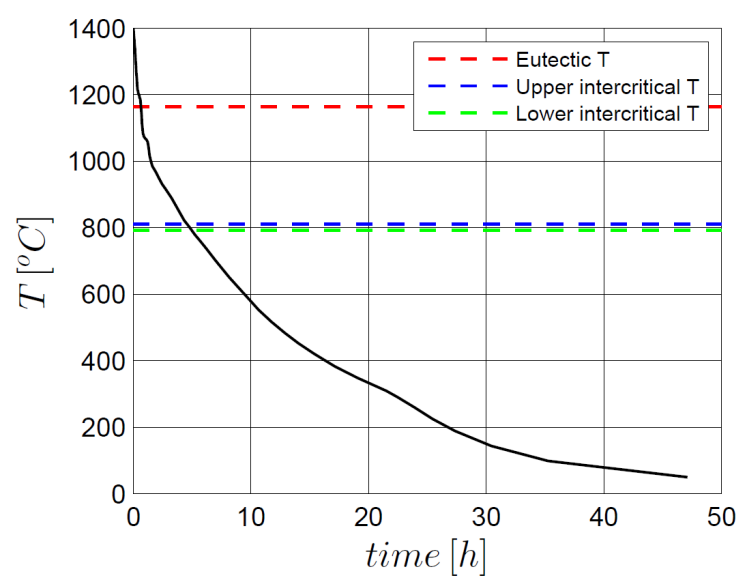

(b)

Figure 6 - Macro-scale modelling. (a) Geometry of the casting with indication of the location from which the DAXM sample was extracted. (b) Numerically predicted cooling curve for the location marked in (a). 


\subsection{Micro-scale level}

To simulate the nodule-matrix interaction during manufacturing, the micro-mechanical model depicted in figure 7 (a) is used. The model is composed of a single spherical nodule surrounded by a matrix shell, whose thickness is adjusted to provide the $11.5 \%$ graphite volume fraction of the SGI under examination. It should be noted that this geometry is consistent with the fact that the nodule considered in the DAXM study (nodule "A" in (Zhang et al., 2016)) had a high spheroidicity level and no close neighboring particles.

The highly-inhomogeneous internal structure of the nodule is discretized explicitly following the physically-based approach initially proposed by (Andriollo et al., 2016a) and subsequently improved by (Andriollo et al., 2016b), which draws on the transmission electron microscopy investigations of (Miao et al., 1990)(Miao et al., 1994)(Monchoux et al., 2001)(Theuwissen et al., 2012)(Theuwissen et al., 2014)(Theuwissen et al., 2016). Accordingly, the nodule is subdivided into $n_{s}=80$ conical sectors radiating from the center of the particle as shown in figure 7 (b). Each sector is subdivided further into a bulk region, composed of graphite platelets oriented perpendicularly to the radial direction, and a superficial layer with thickness $10 \%$ of the nodule radius, characterized by smaller and highly misoriented graphite crystals. Further details and motivations for all these assumptions can be found in (Andriollo et al., 2016b), except for the choice of the number of conical sectors. This last parameter is estimated here from the findings of (Monchoux et al., 2001), who noted that in nodules with diameter $\approx 30 \mu \mathrm{m}$ the sectors are between 2 and 4

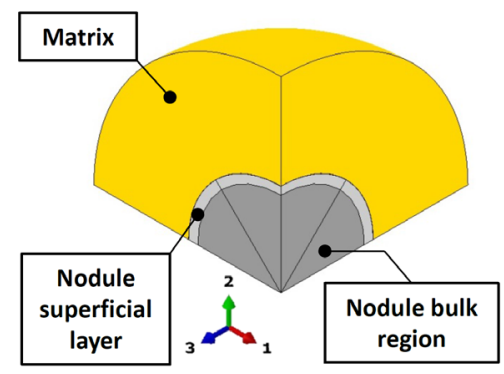

(a)

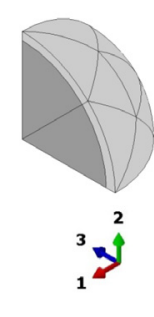

(b)

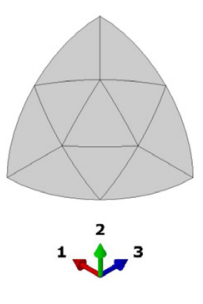

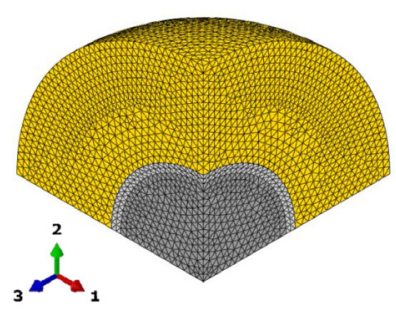

(c)

Figure 7 - Schematic of the micro-scale model. (a) Model geometry with indication of the different microstructural constituents. (b) Close-up view of the subdivision of the nodule into conical sectors. (c) Mesh generated in ABAQUS. 
$\mu \mathrm{m}$ wide at their base. Approximate upper and lower bounds can then be constructed by taking the ratio between the nodule surface area and the basal area of the sectors (assumed circular) as

$$
\frac{4 \pi 15^{2}}{\pi 4^{2}} \approx 56 \leq n_{s} \leq \frac{4 \pi 15^{2}}{\pi 2^{2}}=225
$$

which confirm that 80 is a reasonable assumption.

As done in (Bonora and Ruggiero, 2005), a frictionless contact interface with zero normal tensile strength is assumed to exist between the nodule and the matrix. This is justified by the experimental evidence of the negligible strength of the interface reported in (Berdin et al., 2001). It is worth remarking though, that separate simulations performed assuming a perfect bonding between the nodule and the matrix did not show significant variations in the results. The reason is that mainly normal compressive stresses are transmitted across the interface for the loading scenario analyzed in the present work.

In contrast to the macro-scale level, where all 4 solidification steps described in Section 2.1 are modelled, only the eutectoid transformation and the solid-state cooling in the ferritic field are simulated at the micro-scale. This is because above the upper inter-critical temperature $\mathrm{T}_{\text {ui }}$ the high diffusion rate and the low flow stress of the austenite, in the order of a few MPa only (Kaibyshev and Kazakulov, 2000), are likely to prevent the buildup of significant thermal stresses driven by the CTE mismatch between the nodule and the matrix. Preliminary simulations performed considering the matrix mechanical properties at temperatures above $\mathrm{T}_{\text {ui }}$ equal to those at $\mathrm{T}_{\text {ui }}$, thereby overestimating the matrix strength, confirmed that this assumption is reasonable and affects the final residual stress \& strain field at room temperature to a minor extent only. Consequently, $\mathrm{T}_{\mathrm{ui}}$ is assumed to be the stress-free temperature in the present case. Below $\mathrm{T}_{\text {ui }}$, the temperature of both the nodule and the matrix shell is prescribed according to the cooling curve of figure 6 (b), and it is assumed to be uniform at any time over the entire model volume.

Following (Lacaze and Gerval, 1998), the eutectoid reaction is modelled as a ferrite-halo that nucleates at the graphite-austenite interface and then grows in the radial direction. For simplicity, it is considered that the ferrite fraction in the matrix varies linearly from zero at $\mathrm{T}_{\mathrm{ui}}$ to one at $\mathrm{T}_{\mathrm{li}}$. The local volumetric expansion associated with the austenite-ferrite phase 
transformation is taken equal to $1.2 \%$ (Cockett and Davis, 1963). Furthermore, the nodule radius is assumed to increase linearly by $5 \%$ over the progress of the eutectoid reaction due to carbon diffusion, as discussed in Section 2.1.

The micro-scale model is implemented in the finite element software ABAQUS. Due to symmetry, only $1 / 8$ of the model is discretized, as shown in figure 7 (c). The depicted mesh is constituted of 86970 second-order tetrahedral elements, which are preferred over hexahedral ones to minimize distortion within the conical sectors. The mesh guarantees a numerical error of less than $1 \%$ on the predicted residual elastic strain field at room temperature. Symmetry boundary conditions are applied to the 3 faces orthogonal to the coordinate axes, whereas a uniform radial displacement constraint is imposed on the curved external surface of the matrix shell. The small-strain kinematic framework is adopted and the eutectoid reaction is simulated via the user-defined subroutine UEXPAND. Detailed information about the specific constitutive relations used for the nodule and for the matrix is given in the next section.

\subsection{Constitutive models}

\subsubsection{Matrix}

The constitutive behavior of the matrix is assumed to be described by the isotropic viscoplastic model proposed by (Peric, 1993), which is capable of taking the strain-rate sensitivity pointed out in connection with figure 4 into account. The basic equations of the model are as follows:

- Additive strain decomposition:

$$
\varepsilon^{t o t}=\varepsilon^{e l}+\varepsilon^{v p}+\varepsilon^{t h}
$$

where $\boldsymbol{\varepsilon}^{t o t}, \boldsymbol{\varepsilon}^{e l}, \boldsymbol{\varepsilon}^{v p}, \boldsymbol{\varepsilon}^{t h}$ denote the total, elastic, visco-plastic and thermal strain.

- Linear elastic law:

$$
\boldsymbol{\sigma}=\mathbb{C}^{e l}: \boldsymbol{\varepsilon}^{e l}
$$

where $\boldsymbol{\sigma}$ is the Cauchy stress and $\mathbb{C}^{e l}$ is the $4^{\text {th }}$ order isotropic elastic stiffness tensor.

- Von Mises-type yield function:

$$
\Phi=\sigma_{e}-\sigma_{t h}\left(\varepsilon_{e q}^{v p}\right), \quad \sigma_{e}=\left(\frac{3}{2} \mathbf{s}: \mathbf{s}\right)^{1 / 2}, \quad \mathbf{s}=\boldsymbol{\sigma}-\mathbf{I} \frac{\operatorname{tr}(\boldsymbol{\sigma})}{3}
$$


where $\sigma_{t h}$ is the so-called stress threshold, $\varepsilon_{e q}^{v p}$ is the equivalent visco-plastic strain which obeys the evolution law $\dot{\varepsilon}_{e q}^{v p}=\dot{\lambda}$, I is the $2^{\text {nd }}$ order identity tensor and $\operatorname{tr}(\cdot)$ denotes the trace operator.

- Associative flow rule:

$$
\dot{\boldsymbol{\varepsilon}}^{v p}=\frac{\partial \Phi}{\partial \boldsymbol{\sigma}} \dot{\lambda}, \quad \dot{\lambda}= \begin{cases}\frac{1}{\xi}\left[\left(\frac{\sigma_{e}}{\sigma_{t h}}\right)^{1 / m}-1\right] & \text { if } \Phi\left(\boldsymbol{\sigma}, \sigma_{t h}\right)>0 \\ 0 & \text { if } \Phi\left(\boldsymbol{\sigma}, \sigma_{t h}\right) \leq 0\end{cases}
$$

where $m$ and $\xi$ are the strain-rate sensitivity and the viscosity-related parameter respectively.

To complete the model, the following exponential strain hardening law is selected:

$$
\sigma_{t h}\left(\varepsilon_{e q}^{v p}\right)=R_{0}+\left(R_{\infty}-R_{0}\right) \times\left(1-\exp \left(-\varepsilon_{e q}^{v p} / \varepsilon^{*}\right)\right)
$$

where $R_{0}, R_{\infty}$ and $\varepsilon^{*}$ are material parameters.

The major reason for choosing Peric's model is its behavior in the limit $\sigma_{t h} \rightarrow 0$ and $\xi \rightarrow 0$. Indeed, in the first case the model reduces to the well-known Norton-Hoff model, which is used to describe power-law creep at high temperature. For 1D steady-state conditions, this has the same mathematical structure of equation (1). In the second case instead, the model reduces to the standard rate-independent J2-flow theory of plasticity, which is normally adopted to describe the behavior of metals at room temperature. In such a condition, $\sigma_{t h}$ defines the yield stress evolution, with $R_{0}$ and $R_{\infty}$ representing the initial yield stress $\sigma_{y 0}$ and the ultimate tensile strength $\sigma_{U T S}$ respectively. As a consequence, Peric's model is well suited for the present analysis, where applicability over a wide temperature range is required.

In addition to the standard isotropic elastic moduli and CTE, Peric's model contains 5 additional parameters: 3 related to hardening $\left(R_{0}, R_{\infty}\right.$ and $\left.\varepsilon^{*}\right)$ and 2 related to visco-plastic flow ( $m$ and $\xi$ ), which bring the total number to 8. In this work, Young's modulus is assumed to follow the relation (Schneibel and Heilmaier, 2014)

$$
E(\Delta T)=E_{R T}\left(1+\beta_{R T} \frac{\Delta T}{T_{m}}\right)
$$


where $\beta_{R T}=-0.81, \Delta T$ is the difference between the actual temperature and $20{ }^{\circ} \mathrm{C}$, and $E_{R T}$ and $T_{m}$ are the Young's modulus at $20^{\circ} \mathrm{C}$ and the matrix melting temperature, which are taken equal to $205 \mathrm{GPa}$ and $1773 \mathrm{~K}$ respectively. Furthermore, Poisson's ratio is assumed constant and equal to 0.29 (Schneibel and Heilmaier, 2014), whereas the CTE is provided directly by the curve in figure 5 (a). The remaining model parameters and their temperature dependence are found by means of the identification procedure described in Appendix A, based on the data reported in both figure 4 and in the works referenced in table 2. The identified parameters are shown in figure 8. It is worth noting that at $20{ }^{\circ} \mathrm{C}$ the values of $R_{0}$ and $R_{\infty}$ are quite close to the values of $\sigma_{y 0}$ and $\sigma_{U T S}$ measured by (Baer et al., 1996) on an equivalent matrix material manufactured on the basis of a SGI grade similar to the one considered in the present study. Comparison can also be done between $R_{0}$ and the values of $\sigma_{y 0}$ used by (Bonora and Ruggiero, 2005) in their rate-independent model for ferrite. It is seen that at low temperatures $R_{0}$ is above $\sigma_{y 0}$, while above $\approx 350{ }^{\circ} \mathrm{C}$ the situation reverses, indicating that the present model would allow for visco-plastic flow at lower stress levels.

Before leaving this section, it should be said that Peric's model was implemented as a UMAT subroutine in ABAQUS. For the purpose, the implicit discretization scheme and

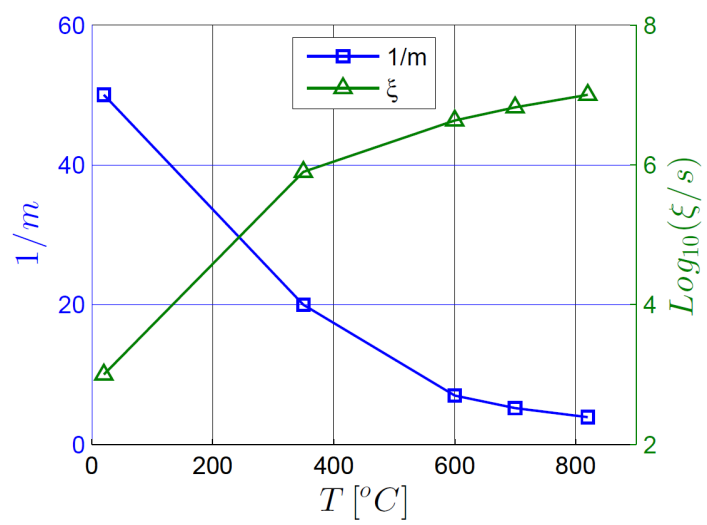

(a)

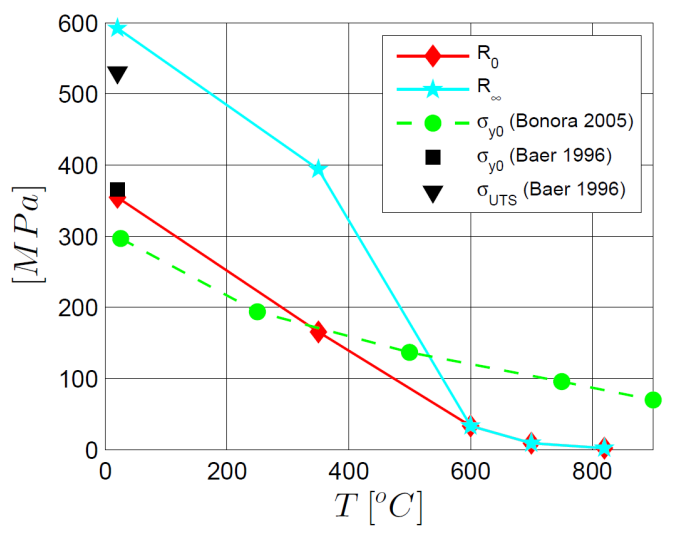

(b)

Figure 8 - Visco-plastic parameters of Peric's model at different temperatures, calibrated via the procedure reported in Appendix A. (a) Strain-rate sensitivity and viscosity-related parameter. (b) Hardening parameters $R_{0}$ and $R_{\infty}$, compared to the values of the initial yield stress $\sigma_{y 0}$ and the ultimate tensile strength $\sigma_{U T S}$ used in (Baer et al., 1996) and in (Bonora and Ruggiero, 2005). 
integration algorithm proposed in (de Souza Neto et al., 2008) were employed. Moreover, the model was assumed to apply to both the ferrite and the austenite within the eutectoid interval, i.e. these two phases were assumed to have the same mechanical behavior in this temperature range.

\subsubsection{Nodule-Elastic case}

The elastic properties of the nodule are assigned following the physically-based approach of (Andriollo et al., 2016b) mentioned in Section 3.2, which guarantees that the roomtemperature elastic behavior of SGI at the macroscale is recovered. Accordingly, the bulk region of the sectors, formed by graphite platelets stacked on each other, is assumed to have the elastic symmetry characteristic of rhombohedral graphite. The associated elastic moduli are reported in table 3 , where the 3 -direction denotes the axis perpendicular to the graphene planes. Such direction is assumed to be oriented, within each conical sector, along the axis connecting the center of the nodule to the centroid of the related spherical triangle, which is formally defined as the element of the nodule surface identified by a given sector. As rhombohedral graphite is transversely isotropic with respect to the basal plane, i.e. the graphene layers, the other two principal material directions can be set arbitrarily without affecting the analysis. The superficial layer of the sectors is assumed to be isotropic instead, with Young's modulus and Poisson's ratio equal to $10 \mathrm{GPa}$ and 0.2 respectively, for the reasons discussed in (Andriollo et al., 2016b). No temperature dependence is taken into account, as this quantity seems to affect the elastic properties of graphite to a limited extent only within the temperature range considered (Faris et al., 1952).

Concerning the thermal contraction, the values proper to graphene are employed for the platelets forming the bulk region of the sectors, whereas the values of the isotropic finegrain graphite IGI-110 are used for the superficial layer. Both sets of data, taken from (Tsang et al., 2005), are plotted in figure 9.

Table 3 - Elastic moduli of rhombohedral graphite (Savini et al., 2011). Entries are in GPa.

\begin{tabular}{ccccc}
\hline C11 & C44 & C12 & C13 & C33 \\
\hline 1107 & 4.4 & 175 & -2.5 & 29 \\
\hline
\end{tabular}




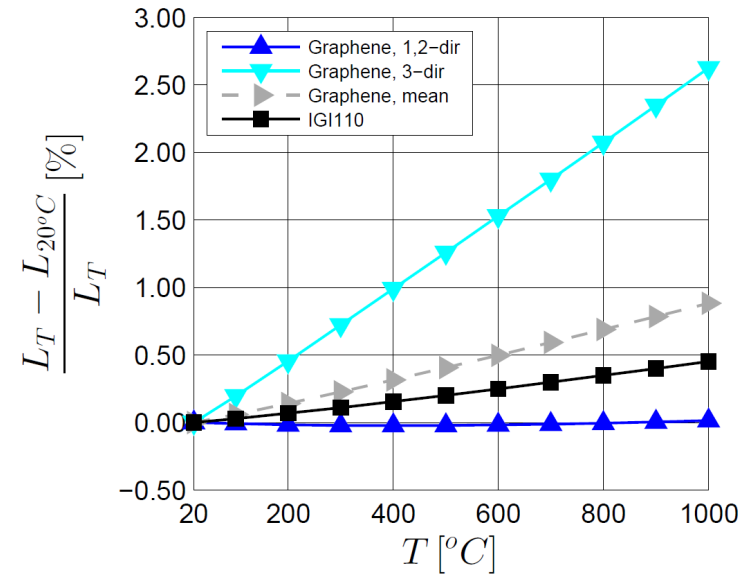

Figure 9 - Engineering thermal strain experienced by graphene and fine-grain isotropic graphite IGI-110 upon cooling from a temperature $\mathrm{T}$ to $20^{\circ} \mathrm{C}$. Source data from (Tsang et al., 2005).

\subsubsection{Nodule-Elastoplastic case}

As will be explained in detail in section 4.1.2, there are indications suggesting that inelastic deformation occurs during manufacturing inside the graphite platelets forming the bulk region of the nodule. Unfortunately, to the authors' best knowledge, dedicated models capable of describing the behavior of the platelets beyond their elastic limit do not exist in the literature. A possible alternative are some models developed in the ' 70 s to describe the elasto-plastic deformation of transversely isotropic types of bulky graphite employed in nuclear applications. In the present study an attempt is made to use the work of (Merkle, 1970), who proposed a rate-independent elasto-plastic model on the basis of the thorough experimental characterization of the transversely isotropic EGCR AGOT graphite reported in (Greenstreet et al., 1969). The model is based on the following ellipsoidal yield function

$$
\begin{gathered}
\Phi(\boldsymbol{\sigma}, \bar{\sigma})=B \sigma_{11}^{2}+B \sigma_{22}^{2}+A \sigma_{33}^{2}+E \sigma_{11} \sigma_{22}+D \sigma_{22} \sigma_{33}+ \\
+D \sigma_{11} \sigma_{33}+(2 B-E) \sigma_{12}^{2}+M \sigma_{13}^{2}+M \sigma_{23}^{2}-\bar{\sigma}^{2}
\end{gathered}
$$

where $\sigma_{i j}, i, j=1,2,3$ are the normal and shear stresses along the principal directions of symmetry, with 3 being the direction perpendicular to the plane of isotropy, and $A, B, D, E, M$ are material parameters whose values are listed in table 4 . The quantity $\bar{\sigma}$ is a power-law hardening function, which has the form

$$
\bar{\sigma}=h_{M}\left(\varepsilon_{e q}^{p}\right)^{r}
$$


Table 4 - Parameters of Merkle's model (Merkle, 1970).

\begin{tabular}{ccccccccc}
\hline $\mathrm{A}$ & $\mathrm{B}$ & $\mathrm{D}$ & $\mathrm{E}$ & $\mathrm{M}$ & $\mathrm{G}$ & $\mathrm{H}$ & $\mathrm{K}$ & $\mathrm{J}$ \\
\hline 1.00 & 2.04 & -0.124 & -0.013 & 0.10 & 1.01 & 0.491 & 0.063 & 0.007 \\
\hline
\end{tabular}

where $h_{M}=315.8 M P a$ and $r=0.44$. The evolution of the equivalent plastic strain $\varepsilon_{e q}^{p}$ is dictated by the expression

$$
\begin{gathered}
\left(\dot{\varepsilon}_{e q}^{p}\right)^{2}=H\left(\dot{\varepsilon}_{11}^{p}\right)^{2}+H\left(\dot{\varepsilon}_{22}^{p}\right)^{2}+G\left(\dot{\varepsilon}_{33}^{p}\right)^{2}+K \dot{\varepsilon}_{11}^{p} \dot{\varepsilon}_{22}^{p}+J \dot{\varepsilon}_{22}^{p} \dot{\varepsilon}_{33}^{p}+J \dot{\varepsilon}_{11}^{p} \dot{\varepsilon}_{33}^{p}+ \\
+\frac{1}{2 B-E}\left(2 \dot{\varepsilon}_{12}^{p}\right)^{2}+\frac{1}{M}\left(2 \dot{\varepsilon}_{23}^{p}\right)^{2}+\frac{1}{M}\left(2 \dot{\varepsilon}_{13}^{p}\right)^{2}
\end{gathered}
$$

where $G, H, J, K$ have the values listed in table 4 and $\dot{\varepsilon}_{i j}^{p}, i, j=1,2,3$ are the plastic strain rates along the principal directions of symmetry. These are quantified by an associative flow rule equivalent to equation (7), with the difference that the plastic multiplier $\dot{\lambda}$ is now determined by the conditions

$$
\Phi \leq 0, \quad \dot{\lambda} \geq 0, \quad \Phi \dot{\lambda}=0
$$

Additive strain decomposition and a linear elastic law of the type shown in equations (4) and (5) complete the model. The elastic moduli of table 3 are assumed to apply. Contrary to Peric's model, suitable implicit discretization and integration schemes had to be devised in order to implement Merkle's model as a UMAT subroutine in ABAQUS. The details can be found in Appendix B.

One limitation of Merkle's model is that it allows for both positive and negative volumetric inelastic deformation. While this is justifiable for nuclear grades of graphite, as they contain a certain fraction of porosity created by the manufacturing process (Merkle, 1970), it is more difficult to rationalize in the case of the bulk region of the nodule, which is fully dense. To compensate for this issue an additional rate-independent elasto-plastic model is adopted in the present study besides Merkle's one, which can still account for the anisotropy and the different tension-compression behavior of the graphite, but produces only isochoric plastic flow. The model rests on the following yield function

$$
\begin{gathered}
\Phi(\boldsymbol{\sigma}, \bar{\sigma})=H_{1}\left(\sigma_{11}-\sigma_{22}\right)^{2}+H_{2}\left(\sigma_{11}-\sigma_{33}\right)^{2}+H_{2}\left(\sigma_{22}-\sigma_{33}\right)^{2}+ \\
+\left(4 H_{1}+2 H_{2}\right) \sigma_{12}^{2}+H_{5}\left(\sigma_{23}^{2}+\sigma_{13}^{2}\right)+H_{7}\left(\sigma_{11}+\sigma_{22}\right)+H_{9} \sigma_{33}-\bar{\sigma}^{2}
\end{gathered}
$$


where the same formalism used in connection with equation (10) applies. Expression (14) represents the particularization to the case of transversely isotropic symmetry of the function proposed by (Hoffman, 1967) to define a fracture criterion for brittle orthotropic materials. Following (de Souza Neto et al., 2008), who dealt with the development of an associative plasticity theory from Hoffman's yield function, the flow rule, additive strain decomposition, linear elastic law and loading/unloading conditions are assumed to have the general format of equations (7), (4), (5) and (13) respectively. Moreover, hardening is considered controlled by the evolution of the von Mises equivalent plastic strain

$$
\dot{\varepsilon}_{e q}^{p}=\left(\frac{2}{3} \dot{\varepsilon}_{i j}^{p} \dot{\varepsilon}_{i j}^{p}\right)^{1 / 2}
$$

where summation over the $\mathrm{i}$ and $\mathrm{j}$ indices is implied. For simplicity, a linear hardening law is postulated in the present work:

$$
\bar{\sigma}\left(\varepsilon_{e q}^{p}\right)=1+h_{H} \varepsilon_{e q}^{p}
$$

As explained in (de Souza Neto et al., 2008), any volumetric plastic flow is inhibited by the constraint

$$
2 \mathrm{H}_{7}+\mathrm{H}_{9}=0
$$

The elastic parameters of the model are assumed to be those reported in table 3 . Moreover, the six parameters controlling the material plastic deformation, i.e. $H_{1}, H_{2}, H_{5}, H_{7}, H_{9}$ and $h_{H}$, are identified using the experimental stress-strain curves of (Greenstreet et al., 1969), obtained by deforming the transversely isotropic EGCR AGOT graphite along its principal directions of symmetry. The details of the procedure are found in Appendix C, whereas the outcome is given in table 5. The model is implemented in ABAQUS as a UMAT subroutine, taking advantage of the implicit discretization and integration schemes presented in (de Souza Neto et al., 2008).

Table 5 - Parameters of Hoffman's model identified using the procedure described in Appendix C.

\begin{tabular}{cccccc}
\hline $\mathrm{H}_{1}\left[\mathrm{MPa}^{-2}\right]$ & $\mathrm{H}_{2}\left[\mathrm{MPa}^{-2}\right]$ & $\mathrm{H}_{5}\left[\mathrm{MPa}^{-2}\right]$ & $\mathrm{H}_{7}\left[\mathrm{MPa}^{-2}\right]$ & $\mathrm{H}_{9}\left[\mathrm{MPa}^{-2}\right]$ & $\mathrm{h}_{\mathrm{H}}$ \\
\hline $2.57 \times 10^{-2}$ & $6.01 \times 10^{-3}$ & $7.30 \times 10^{-2}$ & $-6.22 \times 10^{-3}$ & $1.24 \times 10^{-2}$ & $1.94 \times 10^{2}$ \\
\hline
\end{tabular}




\section{Results and discussion}

\subsection{Predicted VS measured residual elastic strain in the matrix}

This section presents the comparison between the residual elastic strain field predicted by the model and the DAXM measurements shown in figure 1. Initially, the focus is on the peculiar elastic strain pattern seen in proximity to the nodule-matrix interface. After that, the overall gradient and magnitude of the elastic strain at further distance in the matrix is discussed.

\subsubsection{Strain pattern close to the nodule}

To enable comparison with the experimental data, it is necessary to make a choice regarding the positioning of the $\mathrm{XYZ}$ coordinate system of figure 1 with respect to the conical sectors forming the nodule. Indeed, the explicit discretization of the nodule internal structure implies that conditions of spherical symmetry no longer exist in the micromechanical model of figure 7, meaning that any numerical $\varepsilon_{F F}^{e l}$ vs $z$ curve depends on how the origin and the orientation of the XYZ axes are selected. In figure 10 (a) and (b) two possible options are shown, which are denoted by "A" and "B" respectively. The fundamental difference between them relates to the location of the intersection points between the nodule surface and the six paths along which $\varepsilon_{F F}^{e l}$ is recorded. In figure 10 (a) and (b), the paths are sketched as blue dotted lines, whereas the intersection points are marked with blue crosses. It can be seen that, in configuration "A", the origin $\mathrm{P}$ and the orientation of the $\mathrm{XYZ}$ coordinate system are such that the intersection points lie near the boundary between two sectors for the paths closest to P. At the same time, the intersection point lies approximately at the center of a conical sector for the path most distant from P. In configuration " $\mathrm{B}$ " the opposite holds true: the intersection point lies approximately at the center of a conical sector for the path closest to $\mathrm{P}$, and it approaches the boundary with another conical sector when the path distance from $\mathrm{P}$ (quantified by the path $\mathrm{x}$-coordinate) increases. 


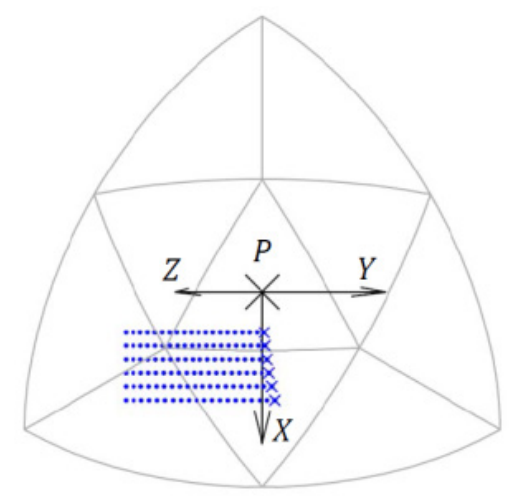

(a)

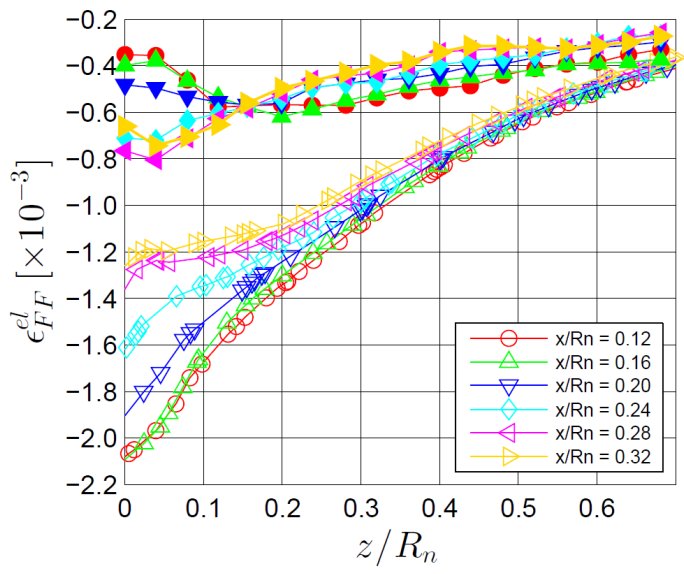

(c)

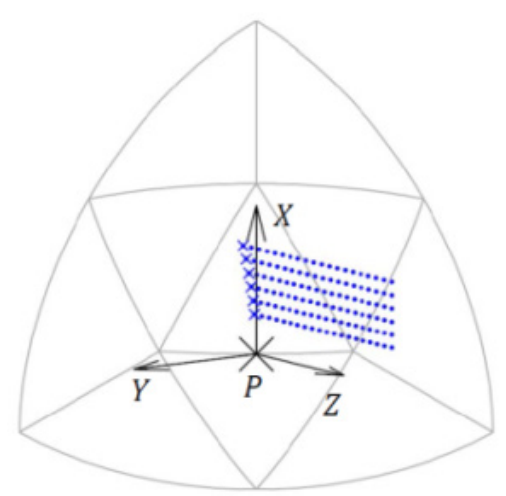

(b)

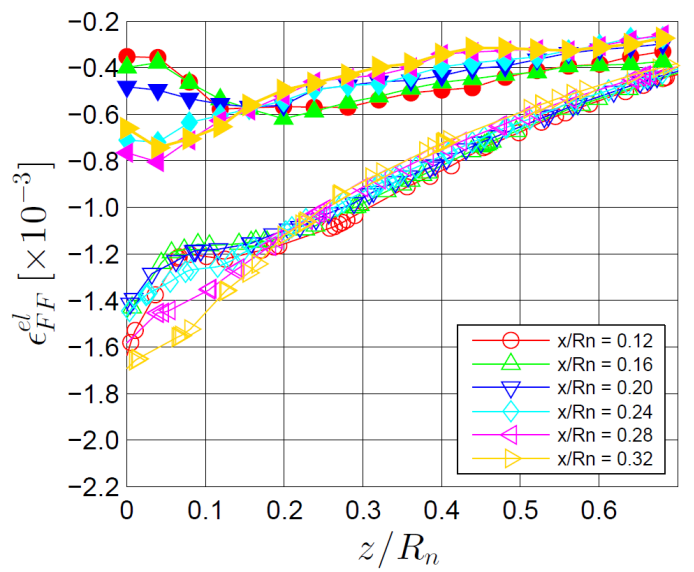

(d)

Figure 10 - Effect of the nodule internal structure on the residual elastic strain field in the matrix. (a), (b) Different configurations of the XYZ coordinate system of figure 1 with respect to the conical sectors forming the nodule, with indication of the six paths along which $\varepsilon_{F F}^{e l}$ is recorded. (c), (d) Comparison between the $\varepsilon_{F F}^{e l}$ vs z curves obtained from DAXM and those extracted from the model according to either the configuration shown in (a) or that shown in (b) respectively. Full markers are used for the experimental curves, empty markers for the numerical ones.

The $\varepsilon_{F F}^{e l}$ vs $z$ curves extracted from the model according to configuration " $\mathrm{A}$ " are shown in figure 10 (c), where they are compared with the DAXM measurements. Besides the overprediction of both the gradient and the maximum magnitude of the residual elastic strain, which will be analyzed in detail in the next section, the shape of the numerical curves deserves special attention. Indeed, it can be observed that, in contrast to the experimental results, the numerical curves never intersect. The strain predicted along a given path is everywhere more compressive (resp. less compressive) than the strain predicted along a 
path which has a higher (resp. lower) value of the $\mathrm{x}$-coordinate, i.e. which is more (resp. less) distant from the origin $\mathrm{P}$ of the XYZ coordinate system. This behavior can be justified on physical grounds. In fact, the greater thermal contraction of the matrix during manufacturing suggests that the material close to the nodule gets stretched in the hoop direction and compressed radially. The direction along which the strain is calculated, defined by the F-axis of figure 1 (a), is almost perfectly aligned with the nodule surface normal when $z, x=0$, meaning that compressive strain values are to be expected at that location. However, when $x$ increases the strain direction deviates progressively more and more from the normal direction. Consequently, the material stretching in the hoop direction starts affecting the calculated strain along the F-axis, which becomes less compressive.

At this point, an obvious question arises as to why the same monotonic, non-intersecting pattern is not seen in the experimental curves as well. One might speculate on the existence of very sharp chemical gradients which, as mentioned in the introduction, could change the value of the unstrained lattice spacing used as reference to calculate the experimental strain. Failure to account for this effect would create artifacts like the intersection point seen in the experimental curves of figure 1 (c). Nevertheless, the gradients required to induce strain variations of up to $5 \times 10^{-4}$ are quite unrealistic, as explained in (Zhang et al., 2016), and in conflict with some experimental investigations which suggest that the radial concentration profiles of the main alloying elements are relatively flat close to the nodule-matrix interface for this grade of SGI (Dong et al., 1997).

A more consistent explanation for the particular pattern of the $\varepsilon_{F F}^{e l}$ vs $z$ curves recorded in the DAXM study can be given on the basis of the residual elastic strain perturbations induced by the conical sectors forming the internal structure of the nodule. These may or may not influence the shape and the monotonicity of the $\varepsilon_{F F}^{e l}$ vs $z$ curves depending on the positioning of the paths along which the strain is recorded with respect to the nodule sectors. In fact, figure 10 (d) shows unequivocally that when configuration " $\mathrm{B}$ " is chosen, the numerical curves predicted by the model intersect and exhibit a pattern which closely resembles the experimental data. The physical reason is that, as stated previously, when the path $\mathrm{x}$-coordinate increases, the nodule-path intersection point gets closer to the boundary between two sectors. Here, the sudden change in orientation of the graphite platelets, 
combined with their high in-plane stiffness, create some local stress concentration. This in turn increases the matrix radial compression at this point compared to the region adjacent to the center of the conical sector, where no stress concentration is present. As a consequence, the strain calculated for the path with $x / R_{n}=0.32$ is, near the nodule, more compressive than that predicted for the paths closer to the origin $\mathrm{P}$, despite the physical argumentation of the previous paragraph would suggest the opposite.

Two examples are useful to obtain further insight into the connection between the nodule internal structure and the residual elastic strain pattern in the matrix. In the first one, the thickness of the superficial layer composing the outer part of the sectors is reduced from 10 $\%$ to $5 \%$ of the nodule radius. Intuition would suggest that by making the isotropic layer thinner, the stress concentration induced in the matrix by the underlying anisotropic graphite platelets would become more pronounced, thereby generating larger perturbations in the strain curves. This is confirmed by the results shown in figure $\mathbf{1 1}$ (a), where it can be seen that the curves spread out twice as much close to the nodule compared to figure $\mathbf{1 0}$ (d). The second example consists of increasing the number of conical sector to infinity to obtain a condition of spherical symmetry. In practice, this can be achieved by prescribing the 3-direction of rhombohedral graphite parallel to the nodule radius pointwise throughout the entire nodule volume. The consequence is twofold: firstly, the positioning of the XYZ coordinate system becomes irrelevant and secondly, the stress concentrations induced by the sector boundaries should disappear. Accordingly, figure 11 (b) indicates that the numerical $\varepsilon_{F F}^{e l}$ vs $z$ curves show a monotonic, non-intersecting behavior over the entire range of $z$ values. It should be recognized that this is also the only pattern that may be generated using a standard, homogeneous isotropic model of the nodule. That is, the intersecting pattern of the experimental curves cannot be reproduced with a model that does not take the internal structure of the nodule into account. This is an additional element that points towards the inadequacy of modeling the nodules as homogeneous isotropic particles, besides those discussed in (Andriollo and Hattel, 2016).

Before concluding this section, it is worth saying that the arguments adduced so far give reason of another experimental fact. In the DAXM investigations, it was shown that the intersecting pattern of the $\varepsilon_{F F}^{e l}$ vs $z$ curves was detected only around 2 out of the 3 nodules 


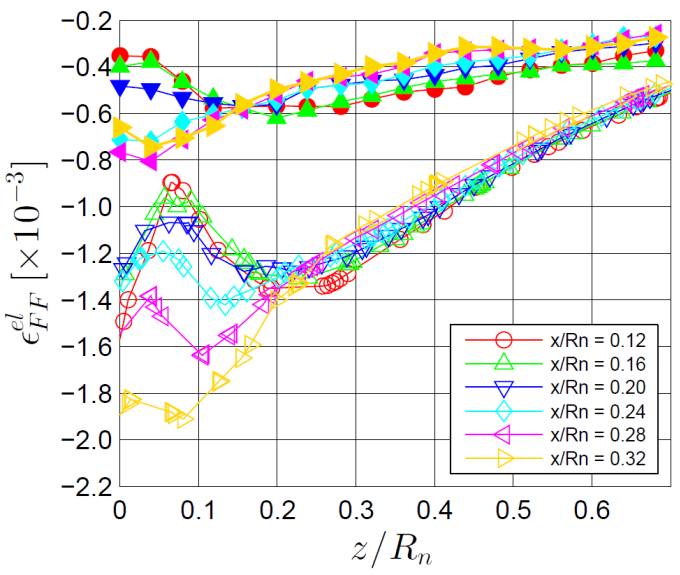

(a)

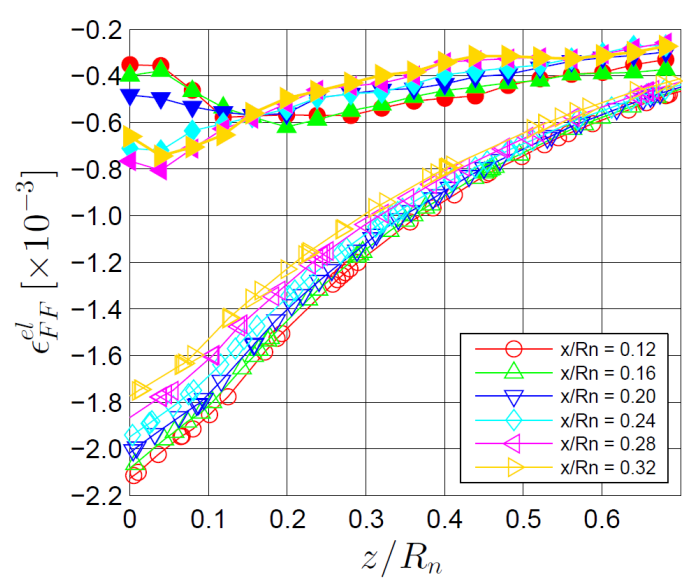

(b)

Figure 11 - Effect of changes in the geometrical parameters describing the nodule internal structure on the predicted residual elastic strain in the matrix. (a) Decrease of the nodule superficial layer thickness from 10 to $5 \%$ of the nodule radius. (b) Increment of the number of conical sectors from 80 to infinite. In both plots, full makers are used for the experimental curves, empty markers for the numerical ones. All the numerical $\varepsilon_{F F}^{e l}$ vs $z$ curves are extracted from the model according to configuration "B" of figure 10 (b).

considered in the study, even though all the nodules came from the same region of the sample (i.e. they had been subjected to the same solidification and cooling conditions). This is coherent with the expected dependence of the shape of the curves on the orientation of the X-ray paths with respect to the sectors forming the nodule, which could not be controlled in the DAXM experiments and therefore may have introduced some degree of randomness in the results.

\subsubsection{Overall strain gradient and magnitude}

The results reported in figure 10 (c) and (d) indicate that the model over-predicts both the overall gradient and the maximum magnitude of the residual elastic strain by a factor $\approx 2$ for $\mathrm{z}>20 \%$ of the nodule radius, no matter the orientation of the XYZ coordinate system with respect to the nodule internal structure. The following paragraphs discuss whether or not this can be explained on the basis of the uncertainty existing on the values of the model parameters.

The geometrical parameters of the micro-mechanical model of figure 7 are considered first. Variations in the thickness of the matrix shell associated with up to $\pm 30 \%$ variation in the 
local graphite concentration are not expected to bring significant changes to the results, for the reasons stated in (Andriollo et al., 2016b). The main source of uncertainty can rather be the number of conical sectors forming the nodule. However, by comparing figure 10 (c) with figure 11 (b) the conclusion is this quantity does not affect the overall strain gradient significantly. Similarly, figure 10 (d) and figure 11 (a) suggest that changes up to $\pm 50 \%$ in the superficial layer thickness are not sufficient to explain the discrepancy with the experimental data for $z$ values greater than $\approx 20 \%$ of the nodule radius.

Concerning the thermo-elastic properties of the constituents, the parameters of the matrix are known to a high level of accuracy. At the same time, the elastic description of the nodule is known to be realistic to the extent that it allows recovering the room-temperature elastic behavior of SGI at the macroscale. The CTE of rhombohedral graphite has been investigated heavily in the literature and the values adopted can be considered as trustworthy. On the contrary, in the present work the CTE of the superficial layer of the nodule is assumed equal to that of IGI-110 isotropic graphite a priori. This could be a source of error, as it is known that isotropic grades of graphite show a large variability in the CTE, between 0.6 and $4.3 \times 10^{-6}{ }^{\circ} \mathrm{C}^{-1}$ at room-temperature (Bonora and Ruggiero, 2005). The main reason seems to lie in the formation of Mrozowski cracks (Hacker et al., 2000), which are nanometric lenticular pores lying parallel to the graphene planes created during manufacture by anisotropic contraction of graphitic crystallites. These prevent achieving the theoretical CTE that would be equal to the mean CTE along the three principal directions of graphene. If this theoretical CTE was used in the model instead of that proper to IGI-110, which is twice as large as seen in figure 9, the thermal expansion mismatch with the matrix should decrease, and the elastic strain gradient should be lowered accordingly. However, figure 12 (a) shows that this effect is, all in all, very limited. 


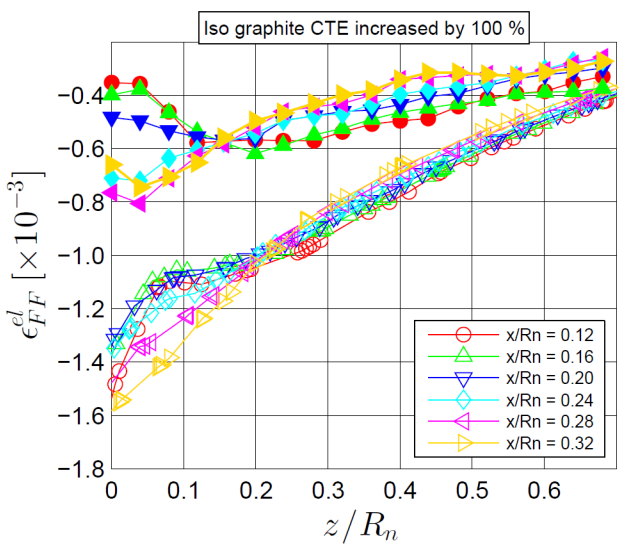

(a)

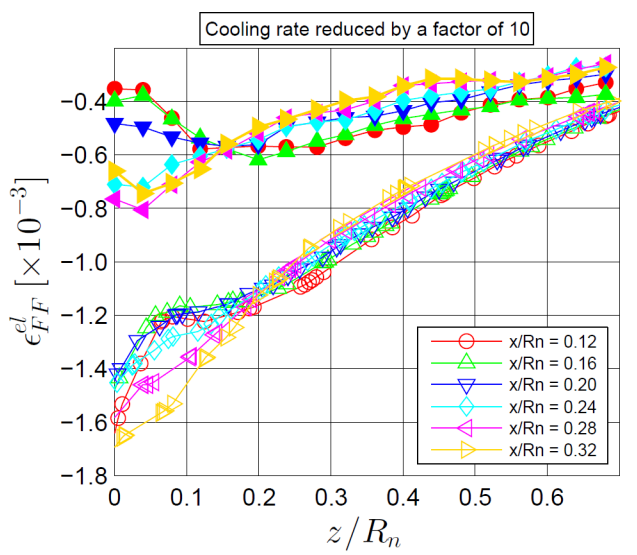

(c)

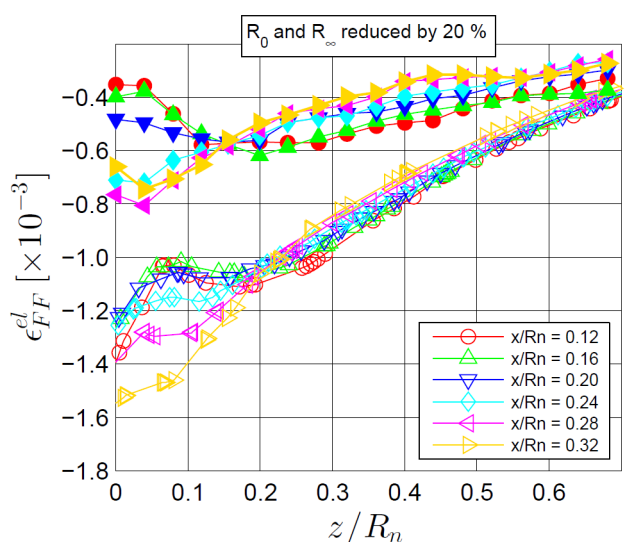

(b)

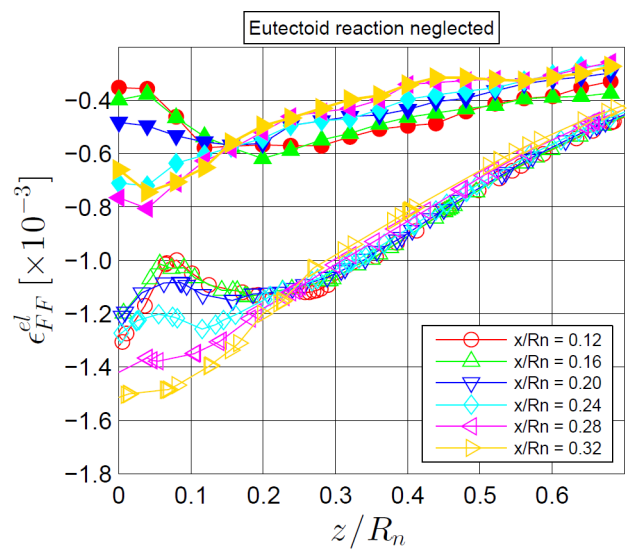

(d)

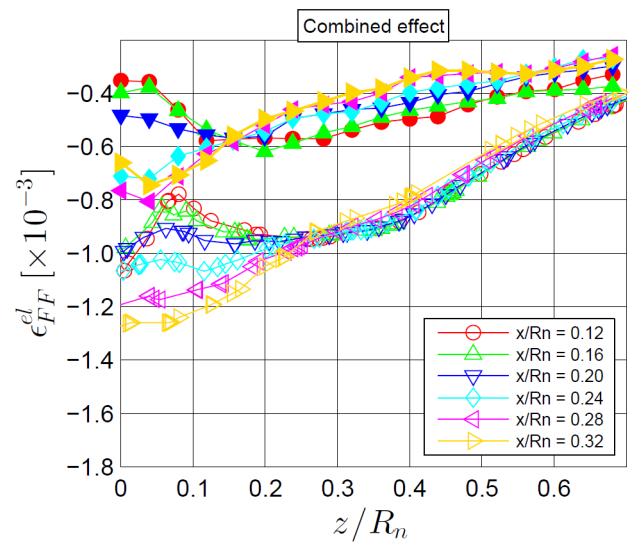

(e)

Figure 12 - Model predictions vs DAXM measurements, under the assumption that significant uncertainty exists on selected model parameters. (a) Isotropic CTE of the superficial layer of the conical sectors increased up to the theoretical value. (b) Hardening parameters $R_{0}$ and $R_{\infty}$ of Peric's model reduced by 20 $\%$ at all temperatures. (c) Cooling rate decreased by a factor of 10 at all temperatures. (d) Eutectoid reaction neglected in the numerical simulations. (e) Effect of (a) to (d) combined. In all plots, full makers are used for the experimental curves, empty markers for the numerical ones. Moreover, all the numerical $\varepsilon_{F F}^{e l}$ vs $z$ curves are extracted from the model according to configuration "B" of figure $\mathbf{1 0}(\mathrm{b})$. 
Given the several assumptions made in the manufacture $\&$ testing of the equivalent matrix material, which in turn affect the calibration of Peric's model, the visco-plastic parameters of the matrix are likely to be affected by a non-negligible error as well. Particularly, it might be that the stress threshold is over-estimated, preventing in this way the achievement of the correct degree of stress relaxation during cooling. Nevertheless, figure 12 (b) indicates that if the values of $R_{0}$ and $R_{\infty}$ are reduced by $20 \%$ at all temperatures, only changes of a few percentages in the elastic strain are produced. As visible in figure 12 (c), an even minor effect is recorded by reducing the cooling rate by a factor of 10 , hence allowing much more time for visco-plastic flow in order to compensate for the uncertainty on both $\xi, m$ and the cooling curve of figure $6(\mathrm{~b})$.

One last aspect which may possibly introduce significant uncertainty in the model is the eutectoid reaction. This is partly due to the simplifying assumptions made in order to simulate its occurrence and partly due to the low accuracy affecting many of the related parameters. To analyze the impact of the eutectoid transformation on the results, it is worth remarking that this reaction creates additional volumetric mismatch between the nodule and the matrix, besides that associated with the standard thermal contraction. Figure 12 (d) shows what happens when the eutectoid reaction is neglected, i.e. the stress free temperature is assumed to be $\mathrm{T}_{\mathrm{li}}$ instead of $\mathrm{T}_{\text {ui }}$. Two observations can be made: first, the magnitude of the elastic strain is not reduced significantly, and second, the curvature of the strain profiles seem to turn upward for $\mathrm{z}$ values between 20 and $40 \%$ of the nodule radius. This last effect is in contrast to the experimental results and therefore suggests that the eutectoid reaction cannot be ignored, even though it does not affect the overall elastic strain magnitude to a great extent.

Finally, figure 12 (e) demonstrates that even by combining the effects of all the most favorable uncertainties analyzed so far, some of which almost unrealistic, it is still not possible to explain the discrepancy with the experimental data in terms of overall residual elastic strain gradient and magnitude. This leads to the conclusion that some fundamental mechanisms and/or physical factors are probably neglected in the model. One of these might be the anisotropy of the matrix grains, which unfortunately cannot be taken into account due to the lack of sufficient crystallographic information regarding the region 
around the nodule. However, the anisotropy of ferrite crystals is not pronounced, therefore the error associated with considering the matrix as isotropic might not be enough to justify the elastic strain over-prediction. Another factor which seems more suited to explain the discrepancy with the experimental data is the inelastic deformation of the nodule, whose occurrence is supported by three facts. First, several studies have detected inelastic deformation and damage of the graphite particles during room-temperature deformation of SGI specimens (He et al., 1997)(Iacoviello et al., 2008)(Di Cocco et al., 2010)(Di Cocco et al., 2014), in some cases even before macroscopic yielding of the matrix. Second, as explained in detail in (Andriollo et al., 2016a) and in (Andriollo et al., 2016b), the nodule carries a hydrostatic load like a rigid shell with high hoop stiffness, due to the circumferential orientation of the graphite platelets forming the bulk region of the conical sectors. This means that the greater contraction of the matrix around the nodule during manufacturing generates large compressive hoop stresses in the latter which lie in the stiff basal planes of the platelets. The formation of twins, bending planes and micro-cracks according to the mechanisms observed by (Freise and Kelly, 1961) in single crystals of graphite is then energetically favorable, as these defects diminish the in-plane length of the platelets thereby decreasing the hoop stresses. Third, the existence of such twins and bending planes within the graphite platelets of different types of cast iron seems to be an experimentally accepted fact (Moumeni et al., 2012)(Moumeni et al., 2014)(Stefanescu et al., 2016). Even though there is no evidence yet that this is linked to the loading scenario just discussed, there is little doubt that it constitutes a valid proof of the occurrence of inelastic deformation within the nodule.

As already stated, no dedicated models have been found in the literature to simulate the inelastic deformation of the graphite platelets. Therefore, two alternatives are considered in the present work. Initially, it is assumed that the platelets deform according to the timeindependent elasto-plastic model of Merkle presented in section 3.3.3, which was developed for transversely isotropic grades of polycrystalline graphite used in nuclear applications. The results obtained using Merkle's model, reported in figure 13 (b), show that the overall gradient and the maximum magnitude of the $\varepsilon_{F F}^{e l}$ vs $z$ curves are reduced by as much as a factor of $\approx 10$ compared to the situation where only the elastic deformation of 
the platelets is considered (figure 10 (c) and (d)). This translates into a factor 5 underestimation of the elastic strain compared to the experimental findings, which are reproduced in figure $\mathbf{1 3}$ (a) for the sake of clarity. This effect is likely a consequence of the irreversible volumetric plastic contraction allowed by Merkle's model, which, as pointed out in section 3.3.3, is difficult to justify from a physical standpoint for a fully dense particle like the nodule. Figure 13 (c) shows what happens when Merkle's model is replaced by Hoffman's model, which is still an associative elasto-plastic model, based on an ellipsoidal yield function and calibrated on the basis of the same type of nuclear graphite, but it allows only isochoric plastic flow. It may be seen that the maximum elastic strain magnitude at $z=0.2 R_{n}$, with $R_{n}$ being the nodule radius, and overall gradient in the interval $0.2 R_{n} \leq z \leq 0.7 R_{n}$ turn out to be overestimated, but only by $20 \%$ and $60 \%$ respectively. It is worth emphasizing that this relatively good agreement is achieved by using material parameters proper to the transversely isotropic EGCR AGOT graphite. Despite the chemical similarity, it is clear that some non-negligible differences compared to the graphite platelets forming the nodule are present, for instance in terms of grain size. One can then hypothesize that if more appropriate material parameters were available, the error on the residual elastic strain magnitude and gradient might be reduced even further. However, it should also be observed that the elastic strain curves of both figure 13 (b) and (c), despite being extracted from the model according to configuration "B", do not exhibit the characteristic intersecting pattern close to the nodule which has been thoroughly analyzed in the previous section. This suggests that associative elasto-plastic models based on ellipsoidal yield functions, like Merkle's or Hoffman's, are actually capable of capturing the behavior of the bulk region of the nodule as a whole, at least to some extent. Nonetheless, they are not effective in describing the local mechanical interactions arising between the single graphite platelets, which strongly affect the residual stress \& strain field in the vicinity of the nodule. 


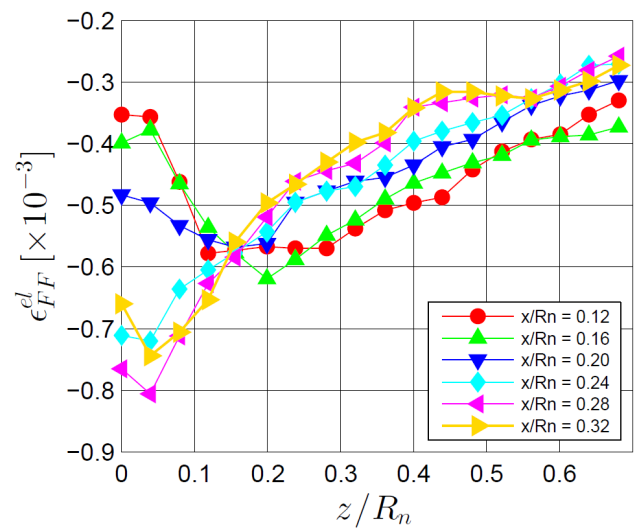

(a)

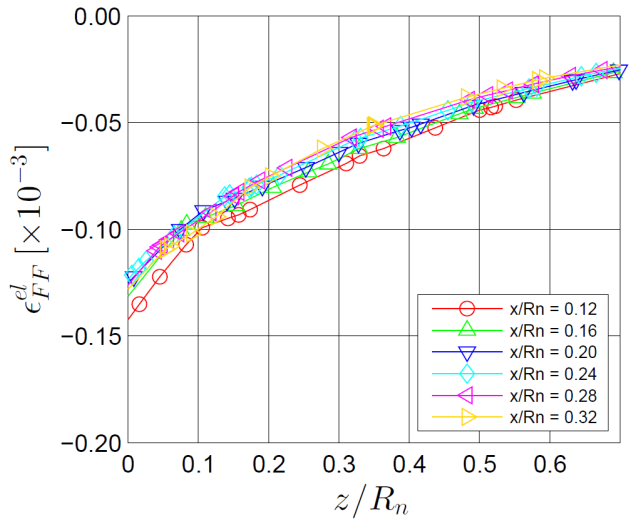

(b)

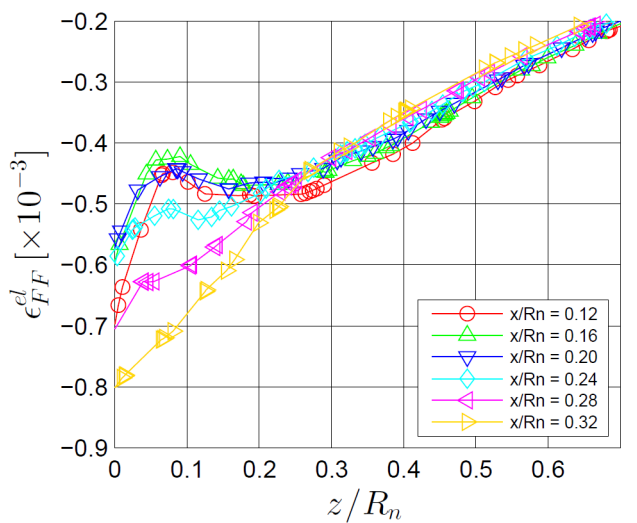

(d)

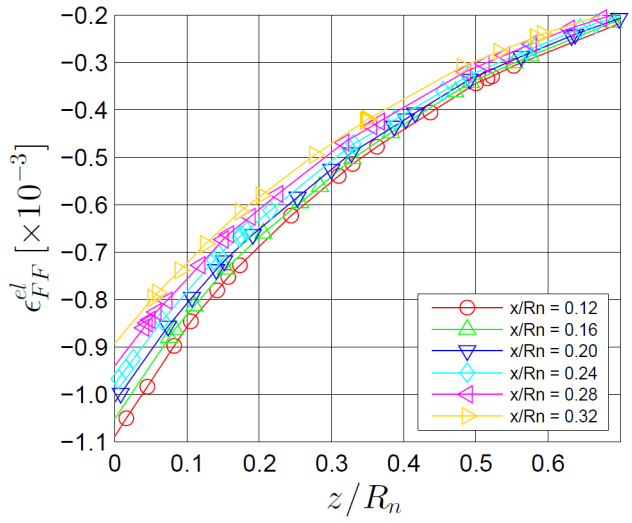

(c)

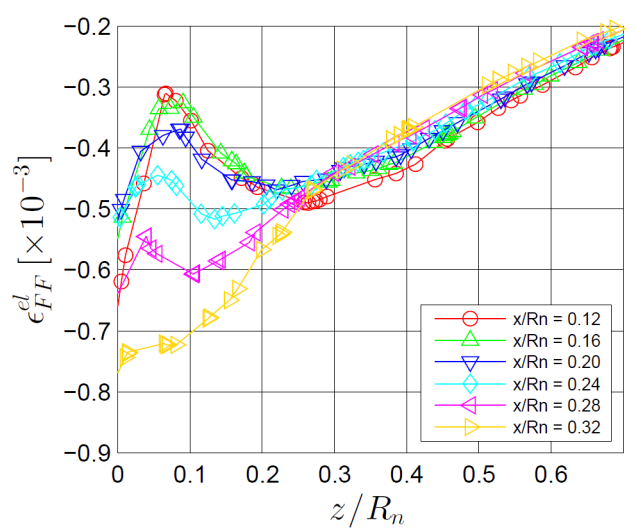

(e)

Figure 13 - Effect of different ways of accounting for the inelastic deformation in the graphite platelets on the predicted residual elastic strain in the matrix. (a) DAXM measurements. (b) Model prediction assuming that the platelets deform elasto-plastically according to Merkle's model. (c) Model prediction assuming that the platelets deform elasto-plastically according to Hoffman's model. (d) Model prediction assuming that the elastic moduli of the platelets are reduced to $15 \%$ of the theoretical values of table 3. (e) The same as in (d), but considering a superficial layer thickness of $5 \%$ of the nodule radius instead of $10 \%$. Note that in (b) - (e) the $\varepsilon_{F F}^{e l}$ vs $z$ curves are extracted from the model according to configuration "B" of figure 10 (b). 
A second, simpler option to simulate the effect of the formation of twins and bending planes within the graphite platelets is to reduce their in-plane stiffness. Indeed, so far their elastic moduli have been assumed to correspond to those of pristine rhombohedral graphite (see table 3), which are valid under the assumption that the material is formed by a perfect stacking of flat graphene layers. On the other hand, recent research has shown that the mechanical properties of graphene are strongly affected by the presence of out-of-plane wrinkles (Baimova et al., 2015)(Qin et al., 2016)(Baimova et al., 2016). Particularly, (Qin et al., 2016) demonstrated that wrinkled graphene sheets with an aspect ratio of 0.15 are characterized by elastic in-plane moduli which are 3-4 times smaller than those exhibited by perfectly flat graphene layers, i.e. with an aspect ratio of zero. In this respect, figure $\mathbf{1 3}$ (d) indicates that a very good agreement with the DAXM measurements is obtained when the in-plane moduli of the graphite platelets are decreased to $15 \%$ of the values given in table 3. The error is of $\approx 15 \%$ on the elastic strain magnitude at $z=0.2 R_{n}$ and of less than $10 \%$ on the overall gradient in the interval $0.2 R_{n} \leq z \leq 0.7 R_{n}$. As visible in figure 13 (e), the agreement with the experimental results can be improved even further, especially in the vicinity of the nodule, by assuming that the thickness of the superficial layer of the particle is $5 \%$ instead of $10 \%$ of the nodule radius. The $5 \%$ value should not look unrealistic, as it lies within the uncertainty range that affects the determination of this parameter (Monchoux et al., 2001). Additional theoretical \& experimental investigations would be required instead to clarify whether or not the twins, bending planes and micro-cracks that have been detected experimentally within the graphite platelets can be responsible for a decrease in the platelet stiffness in the order of $\approx 85 \%$. Such investigations are, however, beyond the scope of the present analysis, due to the supplementary level of theoretical and experimental complexity involved.

\subsection{Predicted residual stress in the matrix}

As mentioned in the last paragraph of the previous section, additional analyses would be necessary to support the second, simple approach used to account for the effect of the inelastic deformation occurring in the graphite platelets during manufacturing. However, even though the underlying physical mechanisms are not simulated in detail, as a matter of 
fact a reduction of the platelet stiffness by a factor 6-7 allows achieving a very good match between model predictions and DAXM measurements in terms of residual elastic strain in the matrix. This result is of crucial importance, as it enables the analysis of the residual stress field existing around the nodule considered in the work of (Zhang et al., 2016), which constitutes the ultimate goal of the present study. Indeed, the linearity of Hooke's law, equation (5), indicates that if the numerical elastic strain field is accurate, the associated stress field can be trusted to a similar level of confidence.

The model predictions in terms of residual stress field in the matrix are shown in figure 14, where the variations of the von Mises, hydrostatic, radial and maximum principal stress are plotted along two different radial paths. These radial paths, which are meant to highlight the effect of the nodule internal structure, are selected as follows. The first one, denoted by "center", intersects the nodule surface at point P of figure 10 (a), i.e. at the center of a conical sector. The second one, denoted by "boundary", intersects the nodule surface at point $\mathrm{P}$ of figure $\mathbf{1 0}$ (b), i.e. at the boundary between two neighboring sectors. Besides giving the results of the model with 80 conical sectors, figure 14 reports also the stress profiles obtained using a nodule with 48 partitions, whose geometry is described in ( Andriollo et al., 2016b), as well as the results corresponding to the limit case of an infinite number of sectors.

It is apparent from figure 14 (a) and (b) that the sector boundary creates some local stress concentration in the neighboring matrix. Indeed, very large deviatoric stresses (von Mises stress of $\approx 330 \mathrm{MPa})$ and moderately high hydrostatic pressure $(\approx 80 \mathrm{MPa})$ are visible at $\mathrm{r} \approx$ 0 for the "boundary" path. Moreover, figure 14 (c) shows that a significant compressive state in the radial direction exists at that location, which supports the arguments adduced in section 4.1 .1 to justify the intersecting pattern of the elastic strain curves of figure $\mathbf{1 0}(\mathrm{d})$. Both the magnitude of the stress concentration and the extent of its propagation into the matrix are enhanced by an increase in the size of the conical sectors. More specifically, increments of $20 \%$ and $100 \%$ in the maximum von Mises and compressive radial stress are recorded when the number of sectors is reduced from infinite to 48 . The associated change in hydrostatic stress is even more pronounced, as the value of this quantity is only a few MPa close to the nodule for the case of an infinite number of sectors. Not surprisingly, 


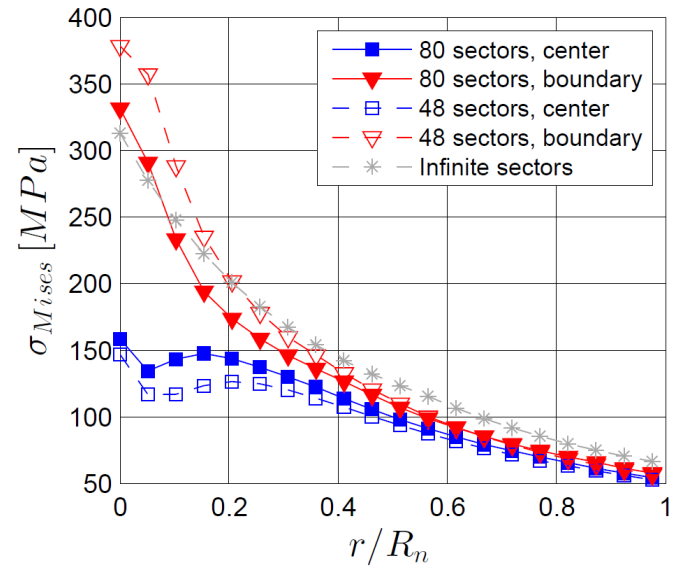

(a)

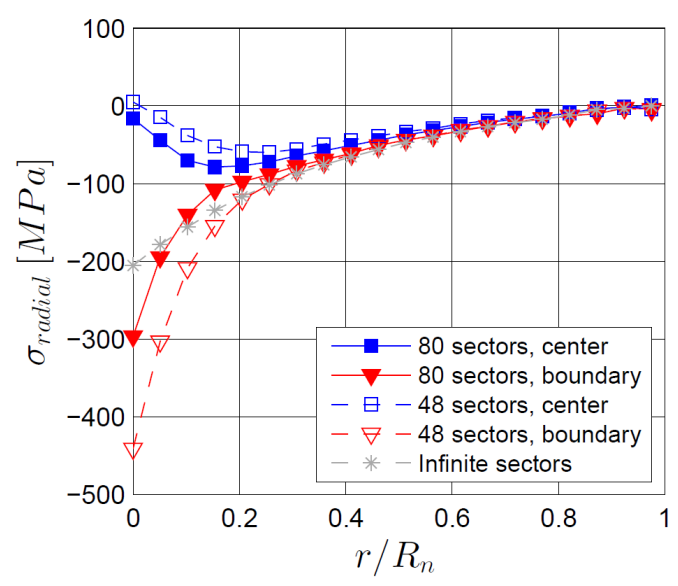

(c)

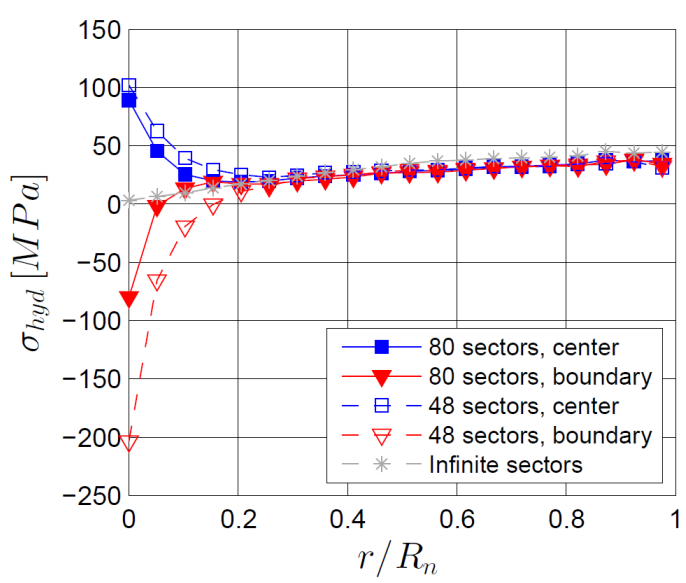

(b)

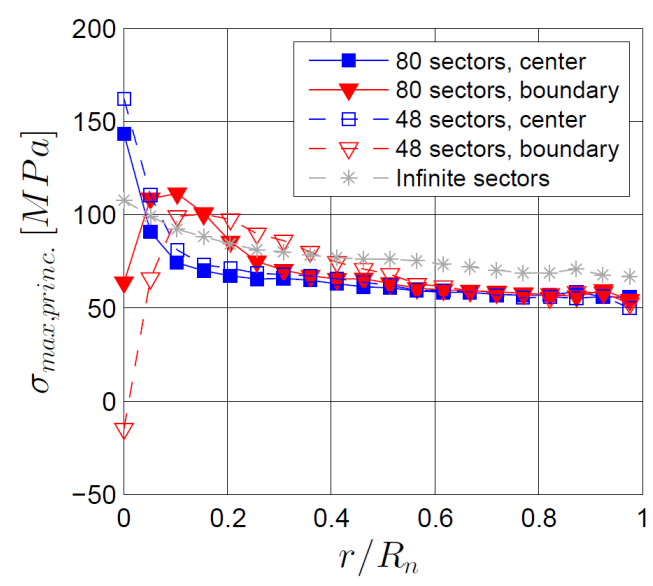

(d)

Figure 14 - Residual stresses in the matrix as functions of the radial distance from the nodule: (a) von Mises stress, (b) hydrostatic stress, (c) radial stress and (d) maximum principal stress. The model parameters are those used to generate the results of figure $13(\mathrm{e})$. Note that the stresses are reported along two different radial paths in the matrix. One, denoted by "center", intersects the nodule surface at point $\mathrm{P}$ of figure 10 (a), i.e. at the center of a conical sector. The other, denoted by "boundary", intersects the nodule surface at point $\mathrm{P}$ of figure 10 (b), i.e. at the boundary between two neighboring sectors.

the stress field perturbation caused by the sector boundary gradually fades away with increasing distance from the nodule, becoming negligible when the latter exceeds $\approx 20$ - 30 $\%$ of the particle radius.

A less intuitive feature of the residual stress field is depicted in figure 14 (d) instead, where it can be seen that, in terms of maximum principal stress, the matrix region adjacent to the center of a sector is more critical than that close to the boundary between two sectors. This 
suggests that while the latter region is likely to deform plastically at some point, the former seems more prone to nucleating a crack, due to the unfavorable combination of positive hydrostatic, low deviatoric and high maximum principal stress.

To conclude this section, is it worth remarking that the maximum stress amplitude that can be applied to the SGI grade under investigation in order to achieve a target fatigue life of 2.5 million cycles under axial loading is $\approx 150 \mathrm{MPa}$ (Shirani and Härkegård, 2011). In addition, the macroscopic $0.2 \%$ proof stress and maximum tensile strength are roughly in the order of $\approx 250 \mathrm{MPa}$ and $400 \mathrm{MPa}$. These values emphasize the impact that the residual stresses of figure 14 may have on the mechanical performance of the SGI component under examination. Consequently, it seems reasonable to conclude that any micro-mechanical fracture model for SGI cannot ignore a priori the existence of such residual stress field by considering the material as stress-free at the micro-scale.

\section{Conclusions}

In the present paper the thermo-mechanical interactions arising between the graphite nodules and the ferrite matrix during manufacturing of SGI were investigated following a combined experimental-numerical approach. First, a material equivalent to the SGI matrix was manufactured and characterized via dilatometry and high-temperature tensile tests. Subsequently, the collected data was used to devise a two-scale model capable of simulating the formation of residual stresses around a nodule placed at a specific location in an industrial SGI component. The results in terms of residual elastic strain in the matrix were compared to DAXM measurements and used to analyze the influence of the nodule internal structure on the shape and magnitude of the residual stress field at the micro-scale. The main findings can be summarized as follows:

1. The equivalent matrix material shows non-negligible strain-rate sensitivity already at $350 \mathrm{C}^{\circ}$. Therefore, rate-dependent constitutive models are necessary to simulate its mechanical response even at temperatures below the eutectoid transformation. On the other hand, the effect of the peculiar silicon content on the material CTE can be ignored. 
2. The characteristic residual elastic strain pattern recorded with DAXM close to the nodule is not an experimental artifact but a real effect. It is caused by the interaction between the matrix and the conical sectors forming the nodule. The impossibility of controlling the positioning of the X-ray beam relative to the sectors introduces a certain degree of randomness in the pattern of the strain profiles recorded with DAXM.

3. When the nodule is assumed to behave elastically, the model overestimates both overall magnitude and gradient of the residual elastic strain in the matrix. The uncertainty existing on the model parameters is not sufficient to explain the discrepancy. The reason is likely the non-negligibility of the irreversible deformation occurring inside the graphite platelets. Associative elasto-plastic theories developed for nuclear grades of graphite can model this in an overall sense, provided that any volumetric inelastic contraction is inhibited, but fail to reproduce the strain pattern recorded close to the nodule. A very good match with the DAXM findings is obtained instead by assuming that the formation of twins and bending planes within the graphite platelets is, all in all, equivalent to a reduction in their elastic stiffness of a factor 6-7 compared to the values proper to graphene.

4. The correct prediction of the residual elastic strain around the nodule provides confidence in using the model to assess the associated stress field. It is concluded that the sector boundaries drive von Mises stress concentrations in the neighboring matrix, whose effect fades away when the distance from the nodule exceeds $\approx 20$ $30 \%$ of its radius. In terms of maximum principal stress though, the matrix region adjacent to the center of a sector seems more critical than that close to the boundary between two sectors. Overall, the maximum residual stresses recorded are comparable with the macroscopic yield stress of the SGI under examination. This indicates that any micro-mechanical fracture model for SGI cannot ignore a priori the existence of the microscopic residual stress field produced during manufacturing. 


\section{Appendix A: Materials parameters identification for Peric's model}

The five material parameters that need to be identified are $R_{0}, R_{\infty}, \varepsilon^{*}, m$ and $\xi$. The strainrate sensitivity parameter $m$ is considered first. In the creep tests reported in the works referenced in table 2 the condition $\left(\sigma_{e} / \sigma_{t h}\right)^{1 / m} \gg 1$ applies. In such a case, Peric's equations reduce to the $1 \mathrm{D}$ expression

$$
\dot{\varepsilon}^{i n}=\frac{1}{\xi \sigma_{t h}^{1 / m}} \sigma^{1 / m}
$$

where $1 / \mathrm{m}$ can be identified as the creep exponent determined from a log-log plot of the stress $\sigma$ and strain rate $\dot{\varepsilon}^{i n}$. The creep exponent is available for all steady-state creep tests mentioned above, and so is $m$. This represents the source of the data plotted in figure 8 (a), with the exception of the room temperature case, where a value of $m=0.02$ is selected which is standard for steels (Tvergaard, 2001).

Uniaxial room-temperature tensile tests performed on the equivalent matrix material at a larger nominal strain rate of $10^{-3} \mathrm{~s}^{-1}$ produced curves overlapping with that given in figure 4, which relates to a nominal strain rate of $10^{-4} \mathrm{~s}^{-1}$. This indicates that the equivalent matrix material strain-rate sensitivity at room-temperature is negligible, at least within this range of deformation rates. Therefore, following (Santos and Rossi, 2013), the classic J2-flow theory of plasticity can be used to identify the hardening parameters $R_{0}, R_{\infty}$, and $\varepsilon^{*}$ (it is worth remarking that, as stated in section 3.3.1, Peric's model reduces to the J2-flow theory in the limit $\xi \rightarrow 0$ ). The identification is made by minimizing the squares of the deviations between the stress measured and the stress predicted by the model for every available time $t_{m}$ and total strain $\varepsilon^{t o t}\left(t_{m}\right)$. The numerical stress at a given time $t_{m}$ is calculated by integration over the experimental pairs $\left(t_{i}, \varepsilon^{t o t}\left(t_{i}\right)\right)$ with $i=0, \ldots, m$, where the total strain is the real one, i.e. the one associated with the deformation experienced by the central cross-section of the specimen. The minimization problem is solved using the genetic algorithm available in Matlab Global Optimization Toolbox (The MathWorks, 2012). The 
identified values of $R_{0}$ and $R_{\infty}$ are plotted in figure $8(\mathrm{~b})$. For $\varepsilon^{*}$, a best-fit value of 0.10 is found, which is assumed to apply at higher temperatures as well.

Concerning the viscoplastic parameter $\xi$, it is assumed to be $1000 \mathrm{~s}$ at room-temperature. This value is similar to those adopted by other authors (de Souza Neto et al., 2008) and it is sufficient to ensure that, at $10 \%$ total strain, the numerical stress predicted during a tensile test at constant $\dot{\varepsilon}^{\text {tot }}=10^{-3} \mathrm{~s}^{-1}$ is only $1 \%$ larger than that calculated using the J2-flow theory. Conversely, at $700{ }^{\circ} \mathrm{C}$ the value of $\xi$ is found by minimizing the model deviation from the corresponding curves of figure 4 (b) by means of the optimization procedure outlined above. In principle, the minimization should be performed considering three unknowns: $\xi, R_{0}$ and $R_{\infty}$. However, one unknown can be eliminated by observing that the intercept $I_{S S}$ of the regression line of figure 4 (c) gives, in light of equation (A.1), the product $\log \left(\xi \sigma_{t h}^{1 / m}\right)$. As the data of figure 4 (c) relate to a total strain of $5 \%$, at which hardening seems to be saturated, the relation

$$
I_{S S}=\log \left(\xi R_{\infty}^{1 / m}\right)
$$

holds true, which provides the constraint needed to eliminate one unknown from the minimization procedure. Finally, at all other temperatures, the viscoplastic parameter $\xi$ is assumed to be described by an Arrhenius-type law:

$$
\xi(T)=\xi_{0} \exp \left(\frac{-Q_{\mu}}{R T}\right)
$$

where $R$ is the universal gas constant and $\xi_{0}=2.92 \times 10^{8} \mathrm{~s}$ and $Q_{\mu}=3.07 \times 10^{4} \mathrm{~J} / \mathrm{mol}$ are parameters, independent of temperature, whose values are calculated by prescribing the already determined values of $\xi$ at room temperature and at $700{ }^{\circ} \mathrm{C}$.

Values for $R_{0}$ and $R_{\infty}$ at $350{ }^{\circ} \mathrm{C}$ and $600{ }^{\circ} \mathrm{C}$ are determined by minimizing the model deviation from the corresponding curves of figure 4 (b) according to the procedure mentioned previously. Moreover, from the results at $700{ }^{\circ} \mathrm{C}$, it can be assumed that $R_{0} \approx R_{\infty}$ above that temperature level. Therefore, values for the former parameters at higher temperatures can be obtained by using equations (A.2) and (A.3) in combination with the intercept $I_{S S}$ of the steady-state creep curves reported in the works of table 2. 


\section{Appendix B: Implicit integration scheme for Merkle's model}

To develop an implicit integration scheme, all the fundamental equations of Merkle's model are reformulated for convenience in matrix form. The yield function, equation (10), is rewritten as

$$
\Phi(\boldsymbol{\sigma}, \bar{\sigma})=\frac{1}{2} \boldsymbol{\sigma}^{\mathrm{T}} \mathbf{P} \boldsymbol{\sigma}-\bar{\sigma}^{2}
$$

where the matrix $\mathbf{P}$ and the stress column vector $\boldsymbol{\sigma}$ are defined as

$$
\mathbf{P}=2 \cdot\left[\begin{array}{cccccc}
B & E / 2 & D / 2 & 0 & 0 & 0 \\
E / 2 & B & D / 2 & 0 & 0 & 0 \\
D / 2 & D / 2 & A & 0 & 0 & 0 \\
0 & 0 & 0 & (2 B-E) & 0 & 0 \\
0 & 0 & 0 & 0 & M & 0 \\
0 & 0 & 0 & 0 & 0 & M
\end{array}\right], \quad \boldsymbol{\sigma}=\left\{\begin{array}{l}
\sigma_{11} \\
\sigma_{22} \\
\sigma_{33} \\
\sigma_{12} \\
\sigma_{13} \\
\sigma_{23}
\end{array}\right\}
$$

The equivalent plastic strain evolution law (12) is recast in the form

$$
\dot{\varepsilon}_{e q}^{p}=\sqrt{\left(\dot{\boldsymbol{\varepsilon}}^{p}\right)^{\mathrm{T}} \mathbf{Z} \dot{\boldsymbol{\varepsilon}}^{p}}
$$

where the matrix $\mathbf{Z}$ and plastic strain rate vector $\dot{\boldsymbol{\varepsilon}}^{p}$ are defined as

$$
\mathbf{Z}=\left[\begin{array}{cccccc}
H & K / 2 & J / 2 & 0 & 0 & 0 \\
K / 2 & H & J / 2 & 0 & 0 & 0 \\
J / 2 & J / 2 & G & 0 & 0 & 0 \\
0 & 0 & 0 & 1 /(2 B-E) & 0 & 0 \\
0 & 0 & 0 & 0 & 1 / M & 0 \\
0 & 0 & 0 & 0 & 0 & 1 / M
\end{array}\right], \quad \dot{\boldsymbol{\varepsilon}}^{p}=\left\{\begin{array}{c}
\dot{\varepsilon}_{11}^{p} \\
\dot{\varepsilon}_{22}^{p} \\
\dot{\varepsilon}_{33}^{p} \\
2 \dot{\varepsilon}_{12}^{p} \\
2 \dot{\varepsilon}_{13}^{p} \\
2 \dot{\varepsilon}_{23}^{p}
\end{array}\right\}
$$

Finally, the flow rule and the linear elastic law are written as

$$
\begin{gathered}
\dot{\boldsymbol{\varepsilon}}^{p}=\dot{\lambda} \mathbf{P} \boldsymbol{\sigma} \\
\boldsymbol{\sigma}=\mathbf{D}^{e l} \boldsymbol{\varepsilon}^{e l}
\end{gathered}
$$

where $\mathbf{D}^{e l}$ denotes the elastic stiffness matrix for transversely isotropic materials. 
If equations (B.1), (B.3), (B.5), (B.6) are first combined with the elastic strain decomposition (4), the hardening law (11) and the loading/unloading conditions (13) and subsequently discretized following the standard backward Euler method, the following system is obtained:

$$
\left\{\begin{array}{l}
\boldsymbol{\varepsilon}_{n+1}^{t o t}-\boldsymbol{\varepsilon}_{n}^{p}-\boldsymbol{\varepsilon}_{n+1}^{t h}=\boldsymbol{\varepsilon}_{n+1}^{e l}+\Delta \lambda \mathbf{P} \boldsymbol{\sigma}_{n+1} \\
\boldsymbol{\sigma}_{n+1}=\mathbf{D}^{e l} \boldsymbol{\varepsilon}_{n+1}^{e l} \\
\Phi_{n+1}=\frac{1}{2} \boldsymbol{\sigma}_{n+1}{ }^{\mathbf{T}} \mathbf{P} \boldsymbol{\sigma}_{n+1}-\bar{\sigma}\left(\varepsilon_{e q, n+1}^{p}\right)^{2} \\
\varepsilon_{e q, n+1}^{p}=\varepsilon_{e q, n}^{p}+\Delta \lambda \sqrt{\left(\mathbf{P} \boldsymbol{\sigma}_{n+1}\right)^{T} \mathbf{Z}\left(\mathbf{P} \boldsymbol{\sigma}_{n+1}\right)} \\
\Phi_{n+1} \leq 0, \quad \Delta \lambda \geq 0, \quad \Phi_{n+1} \Delta \lambda=0
\end{array}\right.
$$

where the subscripts " $n$ " and " $n+1$ " indicate evaluation of the quantity at the beginning and at the end of the time increment respectively, and " $\Delta$ " denotes the corresponding finite variation over the increment. The first of the equations (B.7) can be further multiplied by $\mathbf{D}^{e l}$ and then combined with the second of (B.7) to provide

$$
\boldsymbol{\sigma}_{n+1}^{t r} \equiv \mathbf{D}^{e l}\left(\boldsymbol{\varepsilon}_{n}^{e l}+\Delta \boldsymbol{\varepsilon}^{e l}+\Delta \boldsymbol{\varepsilon}^{p}\right)=\boldsymbol{\sigma}_{n+1}+\Delta \lambda \mathbf{D}^{e l} \mathbf{P} \boldsymbol{\sigma}_{n+1}
$$

The last three of the equations (B.7) in combination with expression (B.8) form a system that can be solved using a standard elastic predictor - plastic corrector algorithm. Indeed, in a finite element software like ABAQUS, both the values of all quantities at the beginning of each increment and $\boldsymbol{\sigma}_{n+1}^{t r}$ are given as input. Initially, it is assumed a priori that $\Delta \lambda=0$ (elastic predictor) and the system of equations is solved trivially. If this does not bring to a violation of the condition $\Phi_{n+1} \leq 0$, the solution is accepted. Otherwise, the following system has to be solved (plastic corrector):

$$
\left\{\begin{array}{l}
\boldsymbol{\sigma}_{n+1}^{t r}-\boldsymbol{\sigma}_{n+1}-\Delta \lambda \mathbf{D}^{e l} \mathbf{P} \boldsymbol{\sigma}_{n+1}=0 \\
\frac{1}{2} \boldsymbol{\sigma}_{n+1}{ }^{\mathbf{T}} \mathbf{P} \boldsymbol{\sigma}_{n+1}-\bar{\sigma}\left(\varepsilon_{e q, n+1}^{p}\right)^{2}=0 \\
\varepsilon_{e q, n+1}^{p}-\varepsilon_{e q, n}^{p}-\Delta \lambda \sqrt{\left(\mathbf{P} \boldsymbol{\sigma}_{n+1}\right)^{T} \mathbf{Z}\left(\mathbf{P} \boldsymbol{\sigma}_{n+1}\right)}=0
\end{array}\right.
$$

This system is equivalent to that analyzed in (de Souza Neto et al., 2008) for the case of Hoffman's model. Therefore, a solution can be found by solving a single non-linear equation according to the procedure documented in the previous reference. 


\section{Appendix C: Materials parameters identification for Hoffman's model}

As already stated in section 3.3.3, the experimental stress-strain curves for the transversely isotropic EGCR AGOT graphite reported in (Greenstreet et al., 1969) are used to calibrate the parameters of Hoffman's model. The quantities $H_{1}, H_{2}, H_{5}, H_{7}, H_{9}$ are determined by imposing the following 5 linear conditions:

- $\Phi(\sigma, 1)=0$ in equation (14) when the $0.1 \%$ proof stress is achieved upon application of a tensile load along direction 3 (perpendicular to the isotropy plane);

- $\Phi(\sigma, 1)=0$ in equation (14) when the $0.1 \%$ proof stress is achieved upon application of a compressive load along direction 3 (perpendicular to the isotropy plane);

- $\Phi(\sigma, 1)=0$ in equation (14) when the $0.1 \%$ proof stress is achieved upon application of a compressive load along direction 1 (in the plane of isotropy);

- $\Phi(\sigma, 1)=0$ in equation (14) when the $0.1 \%$ proof stress is achieved upon shear in the 1-3 plane;

- only isochoric plastic flow is allowed, equation (17).

The parameters determined in this way, listed in table $\mathbf{5}$, satisfy the stability conditions $H_{2} \geq 0$ and $H_{1} \geq-H_{2} / 2$, which ensure that the elastic domain is bounded. It is worth remarking that the use of equation (17) in place of a constraint related to yielding in tension along direction 1 implies that the model predicts a $0.1 \%$ tensile proof stress in the same direction which is $\approx 20 \%$ larger than that measured experimentally. Nevertheless, only compressive stress components develop during manufacturing in the plane parallel to the graphite platelets forming the bulk region of the nodule. Consequently, the platelets are likely to never yield in tension in the 1-2 plane.

The hardening parameter $h_{H}$ is obtained in a second step by imposing the condition $\Phi\left(\sigma, 1+h_{H} \varepsilon_{\max }\right)=0$ in equation (14) when the maximum stress prior to fracture is achieved upon application of a compressive load along either direction 1 or 3 , with $\varepsilon_{\max }$ 
denoting the corresponding strain. Values differing by only $5 \%$ are obtained according to the direction selected. The average value is the one reported in table 5.

\section{Acknowledgements}

Part of this work has been supported by the Strategic Research Center "REWIND Knowledge based engineering for improved reliability of critical wind turbine components,' Danish Research Council for Strategic Research, grant no. 10-093966. The authors gratefully acknowledge Vestas Wind Systems A/S for providing the geometry and the casting details of the wind turbine main shaft used in the simulations.

\section{References}

47th Census of World Casting Production, 2013., MODERN CASTING - A publication of the American Foundry Society.

Andersson, J.-O., Helander, T., Höglund, L., Shi, P., Sundman, B., 2002. Thermo-Calc and DICTRA, computational tools for materials science. Calphad 26, 273-312.

Andriollo, T., Hattel, J., 2016. On the isotropic elastic constants of graphite nodules in ductile cast iron: Analytical and numerical micromechanical investigations. Mech. Mater. 96, 138-150. doi:10.1016/j.mechmat.2016.02.007

Andriollo, T., Thorborg, J., Hattel, J., 2016. Modeling the elastic behavior of ductile cast iron including anisotropy in the graphite nodules. Int. J. Solids Struct. 100-101, 523-535. doi:10.1016/j.ijsolstr.2016.09.023

Andriollo, T., Thorborg, J., Tiedje, N., Hattel, J., 2016. A micro-mechanical analysis of thermo-elastic properties and local residual stresses in ductile iron based on a new anisotropic model for the graphite nodules. Model. Simul. Mater. Sci. Eng. 24, 055012(19pp). doi:10.1088/0965-0393/24/5/055012

Ashby, M., Shercliff, H., Cebon, D., 2010. Materials: engineering, science, processing and design. Butterworth-Heinemann, Oxford, UK.

Baer, W., 2014. Performance of Modern DCI Materials - Investigation of Microstructural, Temperature and Loading Rate Effects on Mechanical and Fracture Mechanical Properties. Mater. Sci. Forum 783-786, 2244-2249. doi:10.4028/www.scientific.net/MSF.783-786.2244

Baer, W., Steglich, D., Brocks, W., Pusch, G., Petit, J., 1996. Experimental determination and micromechanical modelling of damage in nodular cast iron. Proc. 11th Eur. Conf. Fract. (ECF 11).

Baimova, J.A., Korznikova, E.A., Dmitirev, S. V., Liu, B., Zhou, K., 2016. Wrinkles and Wrinklons in Graphene and Graphene Nanoribbons Under Strain. Curr. Nanosci. 12, 184-191. doi:10.2174/1573413711666150523002122

Baimova, J.A., Liu, B., Dmitriev, S. V, Zhou, K., 2015. Mechanical properties of crumpled graphene under hydrostatic and uniaxial compression. J. Phys. D. Appl. Phys. 48, 95302. doi:10.1088/00223727/48/9/095302

Barrett, C., 1967. On stress dependence of high-temperature creep. Trans. Metall. Soc. Aime 239, 1726-1728.

Barrett, C.., Nix, W.., 1965. A model for steady state creep based on the motion of jogged screw dislocations. Acta Metall. 13, 1247-1258. doi:10.1016/0001-6160(65)90034-9

Berdin, C., Dong, M.J., Prioul, C., 2001. Local approach of damage and fracture toughness for nodular cast iron. Eng. Fract. Mech. 68, 1107-1117.

Bonora, N., Ruggiero, A., 2005. Micromechanical modeling of ductile cast iron incorporating damage. Part I: Ferritic ductile cast iron. Int. J. Solids Struct. 42, 1401-1424. doi:10.1016/j.ijsolstr.2004.07.025 
Brown, S.G.R., James, J.D., Spittle, J.A., 1997. A 3D numerical model of the temperature - time characteristics of specimens tested on a Gleeble thermomechanical simulator. Model. Simul. Mater. Sci. Eng. 5, 539-548. doi:10.1088/0965-0393/5/6/001

Cockett, G.H., Davis, C.D., 1963. The lattice expansion of Fe-Si alloys and the volume change at the A3 point. J. Iron Steel Inst. 201, 110-115.

Collini, L., Pirondi, A., 2014. Fatigue crack growth analysis in porous ductile cast iron microstructure. Int. J. Fatigue 62, 258-265. doi:10.1016/j.ijfatigue.2013.06.020

Costa, N., Machado, N., Silva, F.S., 2010. A new method for prediction of nodular cast iron fatigue limit. Int. J. Fatigue 32, 988-995. doi:10.1016/j.ijfatigue.2009.11.005

Davies, R.G., 1963. Steady-state creep in Fe-2 to 11 at. pct Si alloys. Trans. Metall. Soc. Aime 227, 665-668.

de Souza Neto, E., Peric, D., Owens, D.R.J., 2008. Computational methods for plasticity: theory and applications. Wiley.

Di Cocco, V., Iacoviello, F., Cavallini, M., 2010. Damaging micromechanisms characterization of a ferritic ductile cast iron. Eng. Fract. Mech. 77, 2016-2023. doi:10.1016/j.engfracmech.2010.03.037

Di Cocco, V., Iacoviello, F., Rossi, A., Iacoviello, D., 2014. Macro and microscopical approach to the damaging micromechanisms analysis in a ferritic ductile cast iron. Theor. Appl. Fract. Mech. 69, 26-33. doi:10.1016/j.tafmec.2013.11.003

Di Cocco, V., Iacoviello, F., Rossi, a., Cavallini, M., Natali, S., 2013. Graphite nodules and fatigue crack propagation micromechanisms in a ferritic ductile cast iron. Fatigue Fract. Eng. Mater. Struct. 36, 893902. doi:10.1111/ffe. 12056

Dong, M.J., Tie, B., Béranger, A.S., Prioul, C., François, D., 1997. Damage Effect on the Fracture Toughness of Nodular Cast Iron. Adv. Mater. Res. 4-5, 181-188. doi:10.4028/www.scientific.net/AMR.4-5.181

Ductile Iron Society, 2013. Ductile Iron data for design engineers [WWW Document]. URL http://www.ductile.org/ductile-iron-data/ (accessed 10.10.15).

Faris, F.E., Green, L., Smith, C.A., 1952. The thermal dependence of the elastic moduli of polycrystalline graphite. J. Appl. Phys. 23, 89-95.

Fernandino, D.O., Boeri, R., 2015. Study of the fracture of ferritic ductile cast iron under different loading conditions. Fatigue Fract. Eng. Mater. Struct. 38, 610-620. doi:10.1111/ffe.12266

Fernandino, D.O., Cisilino, A.P., Toro, S., Sanchez, P.J., 2017. Multi-scale analysis of the early damage mechanics of ferritized ductile iron. Int. J. Fract. doi:10.1007/s10704-017-0215-1

Freise, E., Kelly, A., 1961. Twinning in graphite. Proc. R. Soc. London Ser. A-mathematical Phys. Sci. 264, 269-276.

Greenstreet, W.L., Smith, J.E., Yahr, G.T., 1969. Mechanical properties of EGCR-type AGOT graphite. Carbon N. Y. 7, 15-45. doi:10.1016/0008-6223(69)90004-9

Grimvall, G., 1997. Cast Iron as a Composite: Conductivities and Elastic Properties. Adv. Mater. Res. 4-5, 31-46. doi:10.4028/www.scientific.net/AMR.4-5.31

Hacker, P.J., Neighbour, G.B., McEnaney, B., 2000. The coefficient of thermal expansion of nuclear graphite with increasing thermal oxidation. J. Phys. D. Appl. Phys. 33, 991-998. doi:10.1088/0022$3727 / 33 / 8 / 316$

He, Z.R., Lin, G.X., Ji, S., 1997. A new understanding on the relation among microstructure micro interfacial mechanical behaviours and macro mechanical properties in cast iron. Mater. Sci. Eng. a 234-236, 161164.

Hoffman, O., 1967. The Brittle Strength of Orthotropic Materials. J. Compos. Mater. 1, 200-206. doi: $10.1177 / 002199836700100210$

Hütter, G., Zybell, L., Kuna, M., 2015a. Micromechanisms of fracture in nodular cast iron: From experimental findings towards modeling strategies - A review. Eng. Fract. Mech. 144, 118-141. doi:10.1016/j.engfracmech.2015.06.042

Hütter, G., Zybell, L., Kuna, M., 2015b. Micromechanical modeling of crack propagation in nodular cast iron with competing ductile and cleavage failure. Eng. Fract. Mech. 147, 388-397. doi:10.1016/j.engfracmech.2015.06.039

Iacoviello, F., Di Bartolomeo, O., Di Cocco, V., Piacente, V., 2008. Damaging micromechanisms in ferriticpearlitic ductile cast irons. Mater. Sci. Eng. A 478, 181-186. doi:10.1016/j.msea.2007.05.110

Iacoviello, F., Di Cocco, V., 2016. Influence of the graphite elements morphology on the fatigue crack propagation mechanisms in a ferritic ductile cast iron. Eng. Fract. Mech. 
doi:10.1016/j.engfracmech.2016.03.041

Kaibyshev, R., Kazakulov, I., 2000. Deformation Behavior of Fe-3\%Si Steel at High Temperatures. Key Eng. Mater. 171-174, 213-218. doi:10.4028/www.scientific.net/KEM.171-174.213

Labrecque, C., Gagne, M., 1998. Review ductile iron: 50 years of continuous development. Can. Metall. Q. $37,343-378$.

Lacaze, J., Gerval, V., 1998. Modelling of the eutectoid reaction in spheroidal graphite Fe-C-Si alloys. Isij Int. $38,714-722$.

Levine, L.E., Okoro, C., Xu, R., 2015. Full elastic strain and stress tensor measurements from individual dislocation cells in copper through-Si vias. IUCrJ 2, 635-642. doi:10.1107/S2052252515015031

Loper Jr., C.R., 1994. The origin of ductile iron - part I. Foundry Manag. Technol. 122, 32-34.

MAGMA GmbH, 2017. MAGMA5 simulation software.

Malagari, F., 1973. Silicon steels and their applications. Met. Eng. Q. 13, 14-18.

Merkle, J.G., 1970. An ellipsoidal yield function for materials that can both dilate and compact inelastically. Nucl. Eng. Des. 12, 425-451. doi:10.1016/0029-5493(70)90056-7

Miao, B., Fang, K., Bian, W., Liu, G., 1990. On the microstructure of graphite spherulites in cast irons by TEM and HREM. Acta Metall. Mater. 38, 2167-2174. doi:10.1016/0956-7151(90)90084-T

Miao, B., North Wood, D.O., Bian, W., Fang, K., Fan, M.H., 1994. Structure and growth of platelets in graphite spherulites in cast iron. J. Mater. Sci. 29, 255-261. doi:10.1007/BF00356601

Monchoux, J.P., Verdu, C., Thollet, G., Fougères, R., Reynaud, A., 2001. Morphological changes of graphite spheroids during heat treatment of ductile cast irons. Acta Mater. 49, 4355-4362. doi:10.1016/S13596454(01)00230-0

Moses, 1990. Electrical steels: past, present and future developments. Phys. Sci. Meas. Instrumentation, Manag. Educ. IEE Proc. a 137, 233-245.

Moumeni, E., Tiedje, N.S., Grumsen, F.B., Danielsen, H.K., Horsewell, A., Hattel, J.H., 2014. A TEM Study on the Ti-Alloyed Grey Iron, in: TMS2014 Annual Meeting Supplemental Proceedings. Minerals, Metals \& Materials Society, San Diego, CA, USA, pp. 943-950. doi:10.1002/9781118889879.ch111

Moumeni, E., Tiedje, N.S., Horsewell, A., Hattel, J.H., 2012. A TEM Study on the Microstructure of Fine Flaky Graphite, in: 52nd International Foundry Conference. Portoroz, Slovenia.

Mukherjee, A.K., 2002. An examination of the constitutive equation for elevated temperature plasticity. Mater. Sci. Eng. A 322, 1-22. doi:10.1016/S0921-5093(01)01115-7

Peric, D., 1993. On a class of constitutive equations in viscoplasticity: formulation and computational issues. Int. J. Numer. Methods Eng. 36, 1365-1393.

Pina, J.C., Kouznetsova, V.G., Geers, M.G.D., 2014. Elevated temperature creep of pearlitic steels: an experimental-numerical approach. Mech. Time-Dependent Mater. 18, 611-631. doi:10.1007/s11043014-9244-6

Qin, H., Sun, Y., Liu, J.Z., Liu, Y., 2016. Mechanical properties of wrinkled graphene generated by topological defects. Carbon N. Y. 108, 204-214. doi:10.1016/j.carbon.2016.07.014

Rivera, G., Boeri, R., Sikora, J., 2002. Revealing and characterising solidification structure of ductile cast iron. Mater. Sci. Technol. 18, 691-697. doi:10.1179/026708302225003668

Salomonsson, K., Olofsson, J., 2017. Analysis of Localized Plastic Strain in Heterogeneous Cast Iron Microstructures Using 3D Finite Element Simulations, in: Mason, P., Fisher, C.R., Glamm, R., Manuel, M. V, Schmitz, G.J., Singh, A.K., Strachan, A. (Eds.), Proceedings of the 4th World Congress on Integrated Computational Materials Engineering (ICME 2017). Springer International Publishing, Cham, pp. 217-225. doi:10.1007/978-3-319-57864-4_20

Santos, T. Dos, Rossi, R., 2013. Calibration of Perzyna-type elasto-viscoplastic models from monotonic unidimensional testing at infinitesimal strains, in: Z.J.G.N Del Prado (Ed.), Proceedings of the XXXIV Iberian Latin-American Congress on Computational Methods in Engineering. Pirenópolis, Brazil.

Savini, G., Dappe, Y.J., Öberg, S., Charlier, J.C., Katsnelson, M.I., Fasolino, A., 2011. Bending modes, elastic constants and mechanical stability of graphitic systems. Carbon N. Y. 49, 62-69. doi:10.1016/j.carbon.2010.08.042

Schneibel, J., Heilmaier, M., 2014. Hall-Petch Breakdown at Elevated Temperatures. Mater. Trans. 55, 4451. doi:10.2320/matertrans.MA201309

Selig, C., Lacaze, A., 2000. Study of microsegregation buildup during solidification of spheroidal graphite cast iron. Metall. Mater. Trans. B 31, 827-836. doi:10.1007/s11663-000-0119-7 
Shirani, M., Härkegård, G., 2011. Large scale axial fatigue testing of ductile cast iron for heavy section wind turbine components. Eng. Fail. Anal. 18, 1496-1510. doi:10.1016/j.engfailanal.2011.05.005

Sjögren, T., Svensson, I.L., 2004. Modelling the effect of graphite morphology on the modulus of elasticity in cast irons. Int. J. Cast Met. Res. 17, 271-279. doi:10.1179/136404604225022694

Smith, W.F., Hashemi, J., 2006. Foundations of Materials Science and Engineering, Fourth. ed. McGraw-Hill.

Sonne, M.R., Thorborg, J., Hattel, J.H., 2017. Modelling the effect of coating on the stresses and microstructure evolution in chill casting of wind turbine main shafts. Wind Energy. doi: $10.1002 /$ we. 2114

Stang, R.G., Nix, W.D., Barrett, C.R., 1973. High temperature creep in Fe-3 pct Si. Metall. Trans. 4, 16951699. doi:10.1007/BF02666198

Stefanescu, D.M., 2017. Mechanical Properties of Ductile Irons, in: Stefanescu, D.M. (Ed.), ASM Handbook, Volume 1A, Cast Iron Science and Technology. ASM International, p. 600.

Stefanescu, D.M., Alonso, G., Larrañaga, P., De la Fuente, E., Suarez, R., 2016. On the crystallization of graphite from liquid iron-carbon-silicon melts. Acta Mater. 107, 102-126. doi:10.1016/j.actamat.2016.01.047

Suits, J.C., Chalmers, B., 1961. Plastic microstrain in silicon-iron. Acta Metall. 9, 854-860. doi:10.1016/0001-6160(61)90189-4

The MathWorks, 2012. MATLAB R2012b.

Theuwissen, K., Lacaze, J., Laffont, L., 2016. Structure of graphite precipitates in cast iron. Carbon N. Y. 96, 1120-1128. doi:10.1016/j.carbon.2015.10.066

Theuwissen, K., Lacaze, J., Véron, M., Laffont, L., 2014. Nano-scale orientation mapping of graphite in cast irons. Mater. Charact. 95, 187-191. doi:10.1016/j.matchar.2014.06.021

Theuwissen, K., Lafont, M.-C., Laffont, L., Viguier, B., Lacaze, J., 2012. Microstructural Characterization of Graphite Spheroids in Ductile Iron. Trans. Indian Inst. Met. 65, 627-631. doi:10.1007/s12666-012$0162-5$

Tiedje, N.S., 2010. Solidification, processing and properties of ductile cast iron. Mater. Sci. Technol. 26, 505514. doi:10.1179/026708310X12668415533649

Tsang, D.K.L., Marsden, B.J., Fok, S.L., Hall, G., 2005. Graphite thermal expansion relationship for different temperature ranges. Carbon N. Y. 43, 2902-2906. doi:10.1016/j.carbon.2005.06.009

Tvergaard, V., 2001. Plasticity and Creep in Structural Materials, Lecture Notes. Danmarks Tekniske Universitet, Kgs. Lyngby.

Vazehrad, S., Elfsberg, J., Diószegi, A., 2015. Study of microstructure and silicon segregation in cast iron using color etching and electron microprobe analysis. Mater. Charact. 104, 132-138. doi:10.1016/j.matchar.2014.09.008

Walser, B., Sherby, O.D., 1982. The Structure dependence of power law creep. Scr. Metall. 16, 213-219. doi:10.1016/0036-9748(82)90389-1

Yang, W., Ice, G.E., Budai, J.D., Tischler, J.Z., Larson, B.C., 2002. Three-dimensional X-ray structural microscopy with submicrometre resolution. Nature 415, 887-890.

Yang, W., Larson, B.C., Tischler, J.Z., Ice, G.E., Budai, J.D., Liu, W., 2004. Differential-aperture X-ray structural microscopy: a submicron-resolution three-dimensional probe of local microstructure and strain. Micron 35, 431-439. doi:10.1016/j.micron.2004.02.004

Zhang, C., Bellet, M., Bobadilla, M., Shen, H., Liu, B., 2010. A Coupled Electrical-Thermal-Mechanical Modeling of Gleeble Tensile Tests for Ultra-High-Strength (UHS) Steel at a High Temperature. Metall. Mater. Trans. A 41, 2304-2317. doi:10.1007/s11661-010-0310-7

Zhang, Y.B., Andriollo, T., Fæster, S., Liu, W., Hattel, J., Barabash, R.I., 2016. Three-dimensional local residual stress and orientation gradients near graphite nodules in ductile cast iron. Acta Mater. 121, 173-180. doi:10.1016/j.actamat.2016.09.009 